\title{
Try-Once-Discard Scheduling for Stochastic Networked Control Systems
}

\author{
Mohammad H. Mamduhi ${ }^{\mathrm{*}}$ and Sandra Hirche ${ }^{\mathrm{a}}$ \\ ${ }^{a}$ Chair of Information-oriented Control, Technical University of Munich, Germany
}

(Received 00 Month 20XX; accepted 00 Month 20XX)

\begin{abstract}
In practical networked control systems (NCS), such as smart grids, cooperative robotics, and sensor networks, often multiple control applications share a communication infrastructure, requiring a smart and efficient scheduling mechanism to coordinate the access to the capacity-limited communication medium. In this article we consider the problem of event-based scheduling design for NCSs consisting of multiple control loops over a shared communication medium. We extend the notion of Try-Once-Discard (TOD), which is one of the basic deterministic event-based scheduling protocols for resource constrained NCSs, to the case of multiple stochastic control systems coupled via a shared communication medium subject to capacity limitation and stochastic packet delivery failure. Showing that the overall network-induced error is a homogeneous Markov chain in our stochastic setup, we first study stability properties of such networked systems under the TOD scheduling scheme employing the concepts of stochastic stability. Then, we derive sufficient stability conditions under the TOD rule assuming that the communication channel is not ideal, i.e., a scheduled data packet for transmission might be lost in the communication channel with a non-zero probability. Furthermore, we derive analytic performance bounds by finding uniform upper-bounds for an average quadratic cost function. The numerical simulations are performed for variety of system parameters and NCS setups to strengthen our stability claim as well as illustrating performance bounds. Additionally, we show that the TOD scheduling rule outperforms the conventional time-triggered, and uniform and non-uniform random channel access arbitration mechanisms, in terms of efficient coordination of channel access in stochastic NCSs.
\end{abstract}

Keywords: Try-Once-Discard (TOD), Markov chain, stochastic stability, network-induced error, performance bound, event-triggered sampling

\section{Introduction}

Classical control theory is typically premised on digital control systems associated with periodic sampling and time-triggered control schemes. The emergence of networked control systems (NCSs) as the integration of multitude of spatially distributed control entities across a shared communication network, however exposed several weaknesses of the traditional control approaches when applied in such NCS setting. The day-by-day increase of data volume which needs to be intelligently exchanged between the distributed parts of a networked system calls for advanced design methodologies and sampling approaches aiming at more efficient usage of costly resources such as energy and bandwidth (R. Gupta \& Chow, 2010; Liu, Wang, He, \& Zhou, 2014). Moreover, control over shared communication channels often imposes challenges ranging from capacity limitations and network congestion to time-varying latency and data loss, which need to be carefully considered (R. Gupta \& Chow, 2010; Hespanha, Naghshtabrizi, \& Xu, 2007; Murray, Åström, Boyd, Brockett, \& Stein, 2003; Zhang, Gao, \& Kaynak, 2013). Numerous works over the last decade show that it is often beneficial to sample the control signals upon occurrence of specific events rather than only after elapsing a fixed temporal period (Åström \& Bernhardsson, 2002; Heemels, Sandee, \& Van Den Bosch, 2008; Henningsson, Johannesson, \& Cervin, 2008; Lunze \& Lehmann, 2010; Tabuada,

*Corresponding author. Email: mh.mamduhi@tum.de 
2007). Further research shows that event-triggered sampling may also be applied successfully on networked systems wherein the control signals are transmitted through a shared communication channel (Al-Areqi, Grges, \& Liu, 2015; Dimarogonas \& Johansson, 2009; Molin \& Hirche, 2013; Ramesh, Sandberg, \& Johansson, 2013; Vilgelm, Mamduhi, Kellerer, \& Hirche, 2016). The aforementioned results suggest that event-based sampling significantly reduces the resource consumption while achieving the same control performance, compared to periodically sampled systems (Cervin \& Henningsson, 2008; Heemels et al., 2008; Molin \& Hirche, 2014a, 2014b; Rabi, Moustakides, \& Baras, 2012; Wang \& Lemmon, 2011).

Most of the available works address the event-trigger synthesis for single-loop networked systems under limited communication resources, where mainly the focus is on generating appropriate event triggers to reduce the transmission rate and consequently communication cost and yet achieve the required control performance including stability (Antunes, Heemels, \& Tabuada, 2012; V. Gupta, Dana, Hespanha, Murray, \& Hassibi, 2009; Lunze \& Lehmann, 2010; McKernan \& Irwin, 2010; Tabuada, 2007). In reality, however, often multiple networked control applications share the communication infrastructure for closing the loop between the sensors and the controllers. Due to the capacity constraints, only a limited number of those loops can be closed at a time. Compared to the single-loop scenario, analysis of multiple-loop NCSs under communication constraints and design of efficient scheduling mechanisms have attained little attention in the literature so far, where some notable exceptions can be found in (Al-Areqi et al., 2015; Blind \& Allgöwer, 2011a, 2011b; Molin \& Hirche, 2014a, 2014b; Ramesh et al., 2013). The results in (Molin \& Hirche, 2014a, 2014b) suggest that the event-based approach can be effectively employed as a threshold policy to govern the channel access in a networked control system with explicitly considered resource limit. It is additionally shown in (Mamduhi, Tolic, \& Hirche, 2015b; Mamduhi, Tolic, Molin, \& Hirche, 2014) that event-triggered scheduling is capable of coping with packet dropouts which is often caused by erroneous network links, malfunctioning network hardware, or environmental disturbances.

Appropriate allocation of scarce communication resources is an active and still challenging research area in the design of NCSs. Attempts have been recently focused on designing the scheduling mechanisms intelligently such that the current situation of the control systems are taken into account when arbitrating the channel access (Mamduhi, Molin, \& Hirche, 2014; Mamduhi, Molin, Tolic, \& Hirche, 2017; Molin \& Hirche, 2014a; Nesic \& Liberzon, 2009; Ramesh et al., 2013; Shi, Epstein, Sinopoli, \& Murray, 2007; Walsh, Ye, \& Bushnell, 2002). Among all, Try-Once-Discard (TOD) is one of the basic event-based scheduling mechanisms which dynamically prioritizes the channel access among the sub-systems requesting for transmission, based on their real-time estimation error (Walsh et al., 2002). Although, TOD is a centralized scheduler and thus not well applicable for large-scale NCSs and multi-hop networks (Christmann, Gotzhein, Siegmund, \& Wirth, 2014), it is an efficient methodology to coordinate the channel access especially for dedicated networks and industrial purposes. For large-scale NCSs and random access networks, distributed and decentralized channel access policies are preferred, (Bauer, Donkers, van de Wouw, \& Heemels, 2013; Gatsis, Ribeiro, \& Pappas, 2015; Mamduhi, Tolic, \& Hirche, 2015a; Wang \& Lemmon, 2011). This topic however is still wide open and is currently under research.

Stability of NCSs under the deterministic TOD protocol is well investigated in the literature and stability conditions are derived in form of lower bounds on the actual inter-transmission intervals. Introducing the concept of Maximal Allowable Transmission Interval (MATI), it is shown that stability is guaranteed if the time difference between two consecutive transmissions is always shorter than sufficiently small MATI (Carnevale, Teel, \& Nesic, 2007; Donkers, Heemels, Bernardini, Bemporad, \& Shneer, 2012; Nesic \& Teel, 2004; Tabbara \& Nesic, 2008; Tabbara, Nesic, \& Teel, 2007; Walsh et al., 2002). In (Walsh et al., 2002), global exponential stability of multiple-packet transmission NCSs consisting of linear time-invariant deterministic systems employing TOD policy is addressed under three assumptions, i.e. the channel is error-free, no observation noise exists, and no model disturbance deteriorates system dynamics. Time-varying transmission intervals and delays, and packet dropouts are later considered in (Donkers et al., 2012; Donkers, Heemels, van de 
Wouw, \& Hetel, 2011), where mean square stability is shown for an NCS of LTI sub-systems with norm-bounded additive uncertainty, orchestrated by a quadratic scheduling protocol of which TOD is shown to be a special realization. The authors of (Nesic \& Teel, 2004; Tabbara et al., 2007) show $\mathcal{L}_{p}$ stability holds for deterministic nonlinear NCSs under TOD with bounded model disturbances, but for sufficiently small MATI. Lyapunov uniform global exponential stability (UGES) for nonlinear deterministic NCSs with exogenous disturbances and stochastic model of dropouts is addressed in (Tabbara \& Nesic, 2008). In (Duc, Christmann, Gotzhein, Siegmund, \& Wirth, 2015), stability of TOD approach is investigated for undisturbed nonlinear NCSs wherein transmissions occur through an unreliable communication channel subject to stochastic communication failure or packet dropouts. In the presence of stochastic noise and exogenous disturbances modeled by continuous random variables, the event triggers, i.e. the network-induced error used in the TOD rule, become stochastic as well. Stochastic event-triggers imply that the channel access is arbitrated randomly in event-based fashion. The concept of MATI however does not apply to stochastic event-triggers and protocols because the time intervals between two consecutive transmissions of a sub-system cannot uniformly be upper-bounded with probability one, therefore calls for employing appropriate stochastic stability concepts. To the best of our knowledge, stability of NCSs composed of multiple stochastically disturbed sub-systems under the event-based TOD scheduling rule with stochastic event-triggers, in the presence of random packet dropouts, is not yet addressed in the literature.

In this article, we study stability and performance of the TOD scheduling rule for NCSs consisting of multiple heterogeneous LTI stochastic controlled sub-systems over shared error-prone communication channel with limited capacity. Each sub-system is disturbed by an independent and identically distributed (i.i.d.) stochastic process selected from the Gaussian distribution with infinite support. Consequently, the local estimation errors, which are considered as the eventtriggers, are driven by the random process. This results in having the transmissions in a biased random fashion. We show that the aggregate network-induced error, which is indeed a stochastic network state, is an aperiodic, irreducible, and homogeneous Markov chain evolving in the uncountable multi-dimensional state-space $\mathbb{R}^{n}$. We show stochastic stability of the resulting multiloop NCS in terms of $f$-ergodicity of the underlying error Markov chain. In addition, we derive the necessary and sufficient condition under which $f$-ergodicity is preserved in case the in-transmission data packets are subject to dropout possibilities through the communication channel. Next, we derive analytic uniform upper-bounds for a given average quadratic cost function. Simulation results validate our stability claims and performance bounds and illustrate the superiority of the TOD approach compared to the related time-triggered, random access, and event-based policies.

In the reminder of this article, Section 2 presents the problem of interest and provides preliminaries regarding stability of stochastic processes. In Section 3, stability of stochastic NCSs under the TOD policy is addressed. Stability results are then extended considering packet dropouts. Performance analysis is presented in Section 4, and numerical results are followed in Section 5. The proofs for the main theorems are brought in details in the Appendix.

Nomenclature: In this article, the Euclidean norm, conditional expectation, and conditional probability are denoted by $\|\cdot\|_{2}, \mathrm{E}[\cdot \cdot \cdot]$, and $\mathrm{P}[\cdot \cdot \cdot]$, respectively. The trace operator is represented by $\operatorname{tr}(\cdot)$. The ceiling operator determines the smallest following integer of a real number, and is denoted by $\lceil\cdot 7$. A random vector $X$ chosen from the multivariate Gaussian distribution with mean vector $\mu$ and covariance matrix $W>0$ is represented by $X \sim \mathcal{N}(\mu, W)$. A sequence of random variables $x_{k}$ evolving in time is shown by $\left\{x_{k}\right\}^{k}$, for all $k \geq 0$. Superscripts and subscripts for state vectors indicate the belonging sub-system, and the time instance, respectively. For matrices though, subscripts indicate the corresponding sub-systems, while a superscript denotes the matrix power. Lastly, we define an augmented vector as $(x, y):=\left[\begin{array}{ll}x^{\top} & y^{\top}\end{array}\right]^{\top}$ to simplify the notation. 


\section{Problem Statement}

The NCS considered in this article consists of $N$ heterogeneous physically independent control loops which are coupled via a shared communication channel subject to capacity limitations. Each control loop $i \in\{1, \ldots, N\}$ is composed of an LTI stochastic process $\mathcal{P}_{i}$ and a control unit including a state estimator and a feedback controller $\mathcal{C}_{i}$, (see Fig. 1). The updated state information from a sub-system is sent for its corresponding control unit if the access to the communication channel is provided. Channel access is governed by a schedule-based medium access controller to resolve the contentions. The process $\mathcal{P}_{i}$ is described by the following LTI discrete stochastic difference equation

$$
x_{k+1}^{i}=A_{i} x_{k}^{i}+B_{i} u_{k}^{i}+w_{k}^{i}, \quad k \geq 0, \quad i \in\{1, \ldots, N\},
$$

where, $x_{k}^{i} \in \mathbb{R}^{n_{i}}, u_{k}^{i} \in \mathbb{R}^{m_{i}}, A_{i} \in \mathbb{R}^{n_{i} \times n_{i}}$ and $B_{i} \in \mathbb{R}^{n_{i} \times m_{i}}$ describe the $i^{\text {th }}$ sub-system state vector, control input, system matrix, and input matrix, respectively. For each sub-system $i$, pair $\left(A_{i}, B_{i}\right)$ is assumed to be controllable. System disturbance $w^{i} \in \mathbb{R}^{n_{i}}$ is presumed to be an i.i.d. random sequence with realization $w_{k}^{i} \sim \mathcal{N}\left(0, W_{i}\right)$, at each time-step $k$. The initial state $x_{0}^{i}$ is supposed to be randomly selected from an arbitrary distribution with bounded second moment. The aggregate initial state $x_{0}$, together with the aggregate stochastic disturbance sequence $w_{k}$, generate the probability space $(\Omega, \mathcal{A}, \mathrm{P})$, where $\Omega$ is the set of all possible outcomes, $\mathcal{A}$ is a $\sigma$-algebra of events with probabilities determined by the function $\mathrm{P}$. At each time-step $k$, the binary variable $\delta_{k}^{i} \in\{0,1\}$ represents the scheduler's decision whether the channel is assigned to a sub-system $i$, as follows

$$
\delta_{k}^{i}= \begin{cases}1, & x_{k}^{i} \text { sent through the channel, } \\ 0, & x_{k}^{i} \text { blocked }\end{cases}
$$

The selected data packets are sent through the communication channel. We assume to have a TCPlike channel, therefore, a successful transmission (i.e., the pertaining packet is not dropped) can be acknowledged, and the acknowledgement signal is represented by the binary variable $\gamma_{k}^{i} \in\{0,1\}$ as

$$
\gamma_{k}^{i}= \begin{cases}1, & x_{k}^{i} \text { successfully received } \\ 0, & x_{k}^{i} \text { dropped }\end{cases}
$$

Based on the scheduling and dropout variables $\delta_{k}^{i}$ and $\gamma_{k}^{i}$, the signal received at the $i^{\text {th }}$ controller is

$$
z_{k}^{i}= \begin{cases}x_{k}^{i}, & \text { if } \delta_{k}^{i} \gamma_{k}^{i}=1 \\ \varnothing, & \text { otherwise }\end{cases}
$$

Each sub-system $i$ is controlled by a state-feedback controller updated at every time-step $k$ by either the true state value $x_{k}^{i}$ (if $\delta_{k}^{i} \gamma_{k}^{i}=1$ ), or by the state estimate $\hat{x}_{k}^{i}$ (if $\delta_{k}^{i} \gamma_{k}^{i}=0$ ). It is assumed that sensor and controller of the $i^{\text {th }}$ sub-system have local knowledge of $A_{i}, B_{i}, W_{i}$ and the distribution of $x_{0}^{i}$. Thus, control law $\vartheta^{i}$ is described by measurable and causal mappings of the past observations:

$$
u_{k}^{i}=\vartheta_{k}^{i}\left(Z_{k}^{i}\right)=-L_{i} \hat{x}_{k}^{i}=-L_{i} \mathrm{E}\left[x_{k}^{i} \mid Z_{k}^{i}\right]
$$

where, $Z_{k}^{i}=\left\{z_{0}^{i}, \ldots, z_{k}^{i}\right\}$ is the $i^{\text {th }}$ controller observation history, and $L_{i}$ is an arbitrary stabilizing feedback gain. In accordance with emulation-based approach, we assume that each loop is stabilized in case of ideal communication, i.e. for $\delta_{k}^{i} \gamma_{k}^{i}=1$ for all time-steps $k \in\{1,2, \ldots\}$. In case of a failed transmission, i.e. $\delta_{k}^{i} \gamma_{k}^{i}=0$, a model-based estimator at the controller side computes the state 
estimate based on the local system parameters $A_{i}, B_{i}$ and $L_{i}$ and state observation history. Thus

$$
\hat{x}_{k}^{i}=\mathrm{E}\left[x_{k}^{i} \mid Z_{k}^{i}\right]= \begin{cases}x_{k}^{i}, & \text { if } \delta_{k}^{i} \gamma_{k}^{i}=1, \\ \left(A_{i}-B_{i} L_{i}\right) \mathrm{E}\left[x_{k-1}^{i} \mid Z_{k-1}^{i}\right], & \text { otherwise }\end{cases}
$$

with $\mathrm{E}\left[x_{0}^{i} \mid Z_{0}^{i}\right]=0$. The estimator (5) is well-behaved since any stabilizing gain $L_{i}$ ensures that the closed-loop matrix $\left(A_{i}-B_{i} L_{i}\right)$ is Hurwitz. Accordingly, we define the one-step ahead networkinduced estimation error $e_{k}^{i} \in \mathbb{R}^{n_{i}}$ as $e_{k}^{i} \triangleq x_{k}^{i}-\mathrm{E}\left[x_{k}^{i} \mid Z_{k-1}^{i}\right]$, with $Z_{k}^{i}=Z_{k-1}^{i} \cup\left\{\delta_{k}^{i}, \gamma_{k}^{i}, \delta_{k}^{i} \gamma_{k}^{i} x_{k}^{i}\right\}$. From (1)-(5), and the definition of $e_{k}^{i}$, the dynamics of the system state and error state are as follows:

$$
\begin{aligned}
& x_{k+1}^{i}=\left(A_{i}-B_{i} L_{i}\right) x_{k}^{i}+\left(1-\theta_{k}^{i}\right) B_{i} L_{i} e_{k}^{i}+w_{k}^{i}, \\
& e_{k+1}^{i}=\left(1-\theta_{k}^{i}\right) A_{i} e_{k}^{i}+w_{k}^{i},
\end{aligned}
$$

where, $\theta_{k}^{i} \triangleq \delta_{k}^{i} \gamma_{k}^{i}$ indicates whether the $i^{\text {th }}$-loop is closed at time $k$. Since various decisions are made within one time period $k$, it is worth specifying the causal ordering of the events, as follows

$$
\cdots \rightarrow x_{k} \rightarrow e_{k} \rightarrow \delta_{k} \rightarrow \gamma_{k} \rightarrow z_{k} \rightarrow u_{k} \rightarrow x_{k+1} \rightarrow e_{k+1} \rightarrow \cdots
$$

This specifies that the scheduler first determines $\delta_{k}$ utilizing the information available at the scheduler at time-step $k$. Afterwards, the error at time-step $k+1$ attains a value according to (7).

It follows from (6) that if the $i^{\text {th }}$-loop is closed at time $k$, i.e. $\theta_{k}^{i}=1$, the stabilizing gain $L_{i}$ ensures the closed-loop matrix $\left(A_{i}-B_{i} L_{i}\right)$ is Hurwitz. We define $\left(x_{k}^{i}, e_{k}^{i}\right)$ as the aggregate state of sub-system $i$. Expression (7) indicates that the evolution of $e_{k}^{i}$ is independent of the system state $x_{k}^{i}$, and control input $u_{k}^{i}$. Therefore, the existence of stabilizing control gains $L_{i}$ does not imply that the network-induced error state $e_{k}^{i}$ is asymptotically converging. In presence of the communication constraints, however, given a stable closed-loop matrix $\left(A_{i}-B_{i} L_{i}\right)$, it is sufficient to show asymptotic convergence of $e_{k}^{i}$ to achieve stability of a sub-system $i$ with the aggregate state $\left(x_{k}^{i}, e_{k}^{i}\right)$. This property enables us to design the scheduler independently from the control law $u_{k}^{i}$.

The scheduling law in this article follows the TOD scheme (Walsh et al., 2002) which is a deterministic and dynamically prioritizing channel access controller. Let the channel capacity be constrained such that not all sub-systems can simultaneously transmit at a time-step, i.e.

$$
\sum_{i=1}^{N} \delta_{k}^{i}=c<N, \quad k \in\{0,1, \ldots\}
$$

where, we assume to have $c$ dedicated channels assuring $c$ parallel transmissions. According to the TOD rule, each sub-system $i$ reports the Euclidean norm of its one-step ahead estimation error at the current time-step $k$, i.e. $\left\|e_{k}^{i}\right\|_{2}$, to the scheduling unit to check if the sub-system is in priority for transmission at that time-step. Having the constraint (8), from all $N$ sub-systems, $c$ sub-systems with the largest error values are selected by the scheduler for transmission, while the other $N-c$ sub-systems are discarded and should try to transmit at next time-steps with updated error values. Having the TOD scheduler described, the transmission decision for sub-system $i$ is taken as follows:

$$
\delta_{k}^{i}= \begin{cases}0, & \text { if }\left\|e_{k}^{i}\right\|_{2}<\left\|e_{k}^{j}\right\|_{2} \text { at least for } c \text { sub-systems } j \neq i \\ 1, & \text { if }\left\|e_{k}^{i}\right\|_{2}^{2}>\left\|e_{k}^{j}\right\|_{2} \text { at least for } N-c \text { sub-systems } j \neq i\end{cases}
$$

In addition, we assume that a data packet which is scheduled for transmission might be dropped out in the communication channel with a non-zero probability. The dropout probability is assumed to follow the Bernoulli distribution. The binary variable $\gamma_{k}^{i} \in\{0,1\}$ represents whether the trans- 


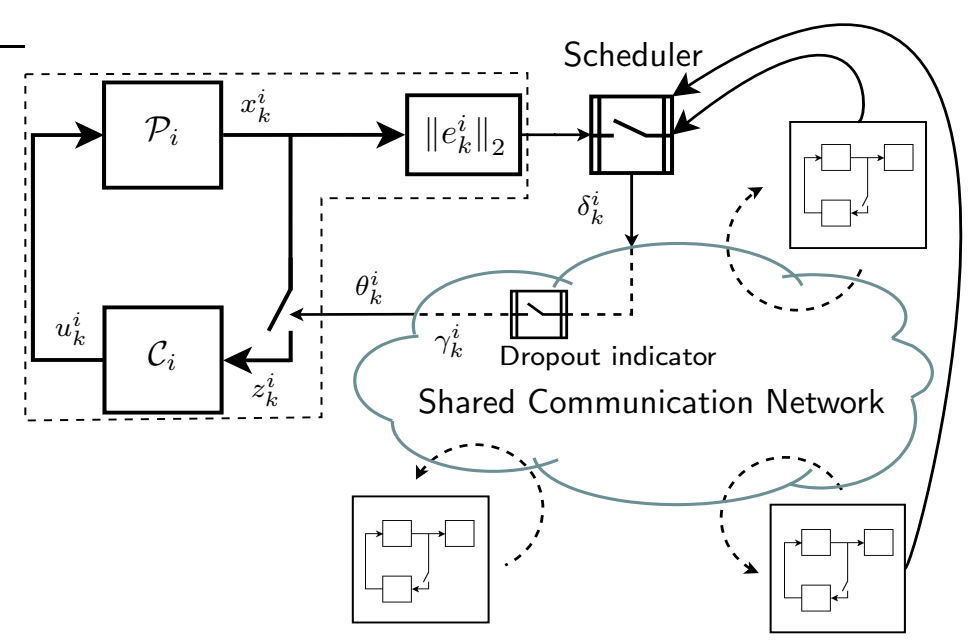

Figure 1. A multi-loop NCS with a shared communication channel and error-dependent scheduler.

mission of a selected sub-system $i$ at time-step $k$, for which $\delta_{k}^{i}=1$, has been successful (see Fig. 1). We comprehensively address the problem of data loss in Section 3.2.1.

In the interest of article brevity, we assume only one sub-system transmits at a time, hence,

$$
\sum_{i=1}^{N} \delta_{k}^{i}=1, \quad \forall k \geq 0
$$

The results of this article can be straightforwardly extended to $\sum_{i=1}^{N} \delta_{k}^{i}=c<N$, where $c>1$.

We define the aggregate one-step ahead estimation error $e_{k} \in \mathbb{R}^{n}=\cup_{i=1}^{N} \mathbb{R}^{n_{i}}$ at time-step $k$ by stacking the local estimation error vectors $e_{k}^{i} \in \mathbb{R}^{n_{i}}$ from all sub-systems $i \in\{1, \ldots, N\}$, i.e.

$$
e_{k}=\left(e_{k}^{1}, \ldots, e_{k}^{N}\right)
$$

According to the expression (7), the sequence $\left\{e_{k}\right\}^{k}$ is controlled by the scheduling variable $\delta_{k}^{i}$ at each time $k$. Moreover, the scheduling law (9) indicates that the decision on $\delta_{k}^{i}$ is made depending only on the most recent one-step ahead estimation error $e_{k}^{i}$. Since $e_{k}^{i}$ 's are noise-driven according to (7), the triggering times of the scheduling events are randomly determined. Moreover, the process noise $w_{k}^{i}$ in (7) has a continuous everywhere-positive density function at any element $e_{k}^{i}$ of the overall state $e_{k}$, i.e., there is a positive probability to reach any subset of $\mathbb{R}^{n}$. This implies the existence of a transition probability $\mathrm{P}$ associated with $\sigma$-algebra $\mathcal{A}$ such that for any event $\mathcal{E} \in \mathcal{A}$

$$
\mathrm{P}\left(e_{k+t} \in \mathcal{E} \mid e_{m}, m<k, e_{k}\right)=P^{t}\left(e_{k+t} \in \mathcal{E} \mid e_{k}\right),
$$

where, $P^{t}\left(e_{k+t} \in \mathcal{E}\right)$ denotes the probability that $e_{k}$ enters a set $\mathcal{E}$ after $t$ transitions, and $m$ is any time index before time $k$. Since the scheduling law (9) does not incorporate the error states $e_{m}$, $m<k$ when deciding on $\delta_{k}^{i}$, and thus the process $\left\{e_{k}\right\}^{k}$ is a $\delta_{k}$-controlled Markov chain. Under each specific binary-valued sequence $\delta_{k}=\left\{\delta_{k}^{1}, \ldots, \delta_{k}^{N}\right\}$, the expression (7) is time-invariant. In addition, the noise realizations $w_{k}^{i}$, s are i.i.d. for each sub-system $i$ at any time $k$ which implies the state transition probability is homogeneous. Moreover, $\delta_{k}$ is $e_{k}$-measurable as it deterministically depends on $e_{k}$, hence, for a given $e_{k}, \delta_{k}$ is known, and the process $\left\{e_{k}\right\}^{k}$ is a homogeneous $\delta_{k}$-controlled Markov chain. Since the noise distribution is absolutely continuous with an everywhere-positive density function, every subset of the state-space is accessible within one transition ( $d$-cycle is one), concluding that $\left\{e_{k}\right\}^{k}$ is an aperiodic and $\psi$-irreducible Markov process, where $\psi$ is the unique maximal irreducibility measure on the $\sigma$-algebra $\mathcal{A}$. Irreducibility guarantees that the entire state space is reachable via a finite number of transitions, regardless of the initial state. 


\subsection{Preliminaries}

Stability analysis of Markov processes in uncountable state-spaces requires additional attention compared to the well-developed stability concepts for those over countable spaces. Stability concept employed in this article is the stochastic notion of $f$-ergodicity. In what follows, we revisit some preliminaries on Markov chains including the concepts of ergodicity, irreducibility, and recurrence.

Definition 1: (Feller, 1971) A random process is called ergodic if the time-average of its events over one sample sequence of transitions represents the process behavior over the entire state-space.

Definition 2: (Hernandez Lerma \& Lasserre, 2001) Let the Markov chain $\Phi=\left(\Phi_{0}, \Phi_{1}, \ldots\right)$ evolve in state-space $\mathcal{X}$, with individual random variables measurable with respect to some known Borel $\sigma$-algebra $\mathcal{B}(\mathcal{X})$. Then $\Phi$ is said to be positive Harris recurrent (PHR) if

(1) There exists a $\sigma$-algebra measure $\nu(B)>0$ for a set $B \in \mathcal{B}$ such that for all initial states $\Phi_{0} \in \mathcal{X}, P\left(\Phi_{k} \in B, k<\infty\right)=1$ holds, where $P(\cdot)$ is the transition probability kernel.

(2) $\Phi$ admits a unique invariant probability measure.

Intuitively, if a state of a PHR Markov chain leaves a subset $B \in \mathcal{B}$ with non-zero probability, then the state returns to the set $B$ after a finite number of transitions, with probability one.

Next, ergodicity of a Markov chain w.r.t. a function $f$, which could be Markov state-dependent, is defined. This is crucial in analyzing the behavior of (7), as the event triggers are error-dependent.

Definition 3: (Meyn \& Tweedie, 1996) Let $f \geq 1$ and $f: \mathbb{R}^{n} \rightarrow \mathbb{R}$. A Markov chain $\Phi$ is said to be $f$-ergodic, if

(1) $\Phi$ is positive Harris recurrent with the unique invariant probability measure $\pi$,

(2) the expectation $\pi(f):=\int f\left(\Phi_{k}\right) \pi\left(d \Phi_{k}\right)$ is finite

(3) $\lim _{k \rightarrow \infty}\left\|P^{k}\left(\Phi_{0}, .\right)-\pi\right\|_{f}=0$ for every initial value $\Phi_{0} \in X$, where $\|\nu\|_{f}=\sup _{|g| \leq f}|\nu(g)|$.

Now we introduce the notion of Markov chain gradient with respect to a real-valued function of states, (Meyn \& Tweedie, 1996). Assume $V: \mathbb{R}^{n} \rightarrow[0,+\infty)$ is a real-valued function and $\Phi$ is a Markov chain. The drift operator $\Delta$ is defined for any non-negative measurable function $V$ as

$$
\Delta V\left(\Phi_{k}\right)=\mathrm{E}\left[V\left(\Phi_{k+1}\right) \mid \Phi_{k}\right]-V\left(\Phi_{k}\right), \quad \Phi_{k} \in \mathbb{R}^{n} .
$$

In the followings, first we define the notion of irreducibility, and then summarizes the notion of $f$-ergodicity for Markov chains in general state-spaces.

Definition 4: (Meyn \& Tweedie, 1996) Let the Markov chain $\Phi$ be defined on $(\mathcal{X}, \mathcal{B})$, where $\mathcal{X}$ is an uncountable space with $\sigma$-algebra $\mathcal{B}(\mathcal{X})$. Let $\varphi$ be a measure on $\mathcal{B}(\mathcal{X})$. Then $\Phi$ is said to be $\varphi$-irreducible, if for every $x \in \mathcal{X}$ and a subset $B \in \mathcal{B}(\mathcal{X}), \varphi(B)>0$ implies $\mathrm{P}\left(\tau_{B}<\infty\right)>0^{1}$. Additionally, If a Markov chain $\Phi$ is $\varphi$-irreducible, then a unique maximal irreducibility measure $\psi \succ \varphi$ exists on $\mathcal{B}(\mathcal{X})$ such that for any $B \in \mathcal{B}(\mathcal{X}), \psi(B)>0$ implies $\mathrm{P}\left(\tau_{B}<\infty\right)>0$.

Theorem 1 ( $f$-Norm Ergodic Theorem): Suppose that the Markov chain $\Phi$ is $\psi$-irreducible and aperiodic and let $f(\Phi) \geq 1$ be a real-valued function in $\mathbb{R}^{n}$. If a petite set $\mathcal{D}$ and a non-negative real-valued function $V$ exists such that $\Delta V(\Phi) \leq-f(\Phi)$ for every $\Phi \in \mathbb{R}^{n} \backslash \mathcal{D}$ and $\Delta V<\infty$ for $\Phi \in \mathcal{D}$, then the Markov chain $\Phi$ is f-ergodic, (Proof in (Meyn $\&$ Tweedie, 1996, Ch. 14)).

${ }^{1}$ Let $\mathcal{X}$ be an uncountable space equipped with $\sigma$-algebra $\mathcal{B}(\mathcal{X})$ and $\Phi=\left(\Phi_{0}, \Phi_{1}, \ldots\right)$ is a Markov chain. Then for any $B \in \mathcal{B}(\mathcal{X})$, the measurable function $\tau_{B}: \Omega \rightarrow \mathbb{Z}^{+} \cup\{\infty\}$ is called return time and denotes the first return time to the set $B$ by $\Phi$, i.e.

$$
\tau_{B}:=\min _{n \geq 1}\left\{\Phi_{n} \in B\right\} .
$$


Remark 1: (Meyn \& Tweedie, 1996) All compact subsets of linear state-spaces are small sets ${ }^{2}$ and a small set is also petite.

In summary, showing a controlled Markov process is $f$-ergodic confirms that the Markov state evolves according to an invariant finite-variance measure over entire state-space. This ensures that the Markov chain is a stationary process, and guarantees if the Markov state leaves some subsets of $\sigma$-algebra $\mathcal{B}(\mathcal{X})$, it can be steered to return to these subsets in finite time with probability one.

Remark 2: For the sake of simplicity in this article, we assume that the measurements, that are communicated to the scheduler and controllers, are perfect copies of state vectors $x_{k}^{i}$ 's, without additive measurement noise. The results of this work are repeatable in case of having noisy measurements presuming the observability condition, and employing Kalman filters and state observers at each controller side. It is worth noting that stochastic measurement noise invalidates the Markov property of the aggregate error state $e_{k}$. Therefore, appropriate alternatives, e.g. Lyapunov mean square stability, need to be employed for stability analysis, (Mamduhi, Kneissl, \& Hirche, 2016).

\section{Stability Analysis}

In this section, we study stochastic stability of multiple-loop NCSs described in (1)-(7) over shared communication channels subject to the constraint (10), under the event-based TOD scheduling policy (9). As previously discussed, assuming that the emulation-based control laws are pre-designed according to (4), the overall NCS stability will be achieved if the overall network-induced estimation error $e_{k}$ is stationary. To that end, we employ Theorem 1 to analyze dynamics of the $\delta_{k}$-controlled Markov process $\left\{e_{k}\right\}^{k} \in \mathbb{R}^{n}$. We first select the following non-negative function $V: \mathbb{R}^{n} \rightarrow \mathbb{R}_{\geq 0}$ :

$$
V\left(e_{k}\right)=\sum_{i=1}^{N} e_{k}^{i^{\top}} e_{k}^{i}=\sum_{i=1}^{N}\left\|e_{k}^{i}\right\|_{2}^{2}
$$

Due to the characteristics of the selected function (13) and the capacity constraint (10), $f$-ergodicity of the Markov process $\left\{e_{k}\right\}^{k}$ cannot always be guaranteed independent of initial conditions, for general parameters $A_{i}, W_{i}$, and $c$, employing the drift $\Delta V$ in (12) defined over one transition step. We illustrate this observation by computing the one-step drift (12) for a descriptive NCS setup.

Example 1: Let two identical scalar LTI sub-systems compete for the sole shared channel slot at each sampling time $k$. For the sake of illustrative purposes, assume that the communication channel is perfect, i.e. no packet drop out occurs. At an arbitrary time-step $k$, let $\left|e_{k}^{1}\right|<\left|e_{k}^{2}\right|$, therefore, $\delta_{k}^{2}=1$ according to the TOD rule (9), and consequently from (7) we have $e_{k+1}^{2}=w_{k}^{2}$. Employing (13) with $e_{k}=\left(e_{k}^{1}, e_{k}^{2}\right)$, the drift operator (12) yields the following:

$$
\begin{aligned}
\Delta V\left(e_{k}\right) & =\mathrm{E}\left[V\left(e_{k+1}\right) \mid e_{k}\right]-V\left(e_{k}\right) \\
& =\mathrm{E}\left[\left|e_{k+1}^{1}\right|^{2}+\left|e_{k+1}^{2}\right|^{2} \mid e_{k}^{1}, e_{k}^{2}\right]-\left|e_{k}^{1}\right|^{2}-\left|e_{k}^{2}\right|^{2} \\
& =\mathrm{E}\left[\left|A_{1} e_{k}^{1}+w_{k}^{1}\right|^{2} \mid e_{k}^{1}\right]+\mathrm{E}\left[\left|w_{k}^{2}\right|^{2}\right]-\left|e_{k}^{1}\right|^{2}-\left|e_{k}^{2}\right|^{2} \\
& =\left|A_{1}\right|^{2}\left|e_{k}^{1}\right|^{2}+W_{1}+W_{2}-\left|e_{k}^{1}\right|^{2}-\left|e_{k}^{2}\right|^{2}=\left(\left|A_{1}\right|^{2}-1\right)\left|e_{k}^{1}\right|^{2}+W_{1}+W_{2}-\left|e_{k}^{2}\right|^{2}
\end{aligned}
$$

which is not always negative for general $A_{1}, W_{1}$, and $W_{2}$, hence violating the ergodicity condition in Theorem 1. In fact the drift might be negative only for certain initial conditions $e_{k}^{1}$ and $e_{k}^{2}$, but not generally. As Theorem 1 provides only sufficient ergodicity condition for a Markov process, a positive drift does not ensure that $\left\{e_{k}\right\}^{k}$ is not ergodic. Therefore, as we will discuss it in the following section, with employing an alternative approach we may still certify ergodicity.

${ }^{2}$ A subset $C \in \mathcal{B}(\mathcal{X})$ of the measurable space $(\mathcal{X}, \mathcal{B})$ is called $\nu$-small if a non-trivial measure $\nu$ on $\mathcal{B}(\mathcal{X})$ and $k>0$ exists such that for all $x \in C$, and $B \in \mathcal{B}(\mathcal{X}), P^{k}(x, B) \geq \nu(B)$ holds. 


\subsection{Stability analysis using multi-step drift}

we aim to analyze the behavior of the overall networked system under the TOD scheduling law, which is an online medium access controller. This emphasizes that the current situation of each subsystem is decisive in the eventual transmission order. Note that, noise realization $w_{k}^{i}$, in addition to the system parameter $A_{i}$, affects the triggering law (9). Therefore, internally stable sub-systems may transmit ahead of unstable ones due to having a large noise realization. It is then theoretically possible that an unstable sub-system has to wait for multiples of time-steps to transmit. Therefore, considering only a one-time-step transition to verify stability might result in conservative conditions. To take this stochastic effect into account, we propose a less conservative stability test which employs the drift criterion over an interval of multiple time-steps. This time interval, over which the drift operator $\Delta V$ is defined, guarantees that all sub-systems in the NCS have non-zero probabilities of having at least one transmission. It should however be noted that, non-zero probability of transmission never guarantees an eventual transmission for a certain sub-system. Depending on the number of sub-systems $N$, and the channel capacity $c<N$, we first derive the minimumlength of such time interval, and then discuss that convergence of the Markov process $\left\{e_{k}\right\}^{k}$ over shorter intervals will only be achieved via conservative conditions on initial conditions and system parameters. Before that, we define the multi-step drift operator over an interval $[k, k+l]$, as follows:

$$
\Delta V\left(e_{k}, l\right)=\mathrm{E}\left[V\left(e_{k+l}\right) \mid e_{k}\right]-V\left(e_{k}\right), e_{k} \in \mathbb{R}^{n} .
$$

To ensure convergence of the Markov chain $\left\{e_{k}\right\}^{k}$ over an interval, a Lyapunov candidate $V\left(e_{k}\right)$ needs to be always drifting inward, i.e. the drift is negative over the considered time interval. Consider $\frac{N}{c}>1$, which implies the integer $\left\lceil\frac{N}{c}\right\rceil \geq 2$. Let the Markov process $\left\{e_{k}\right\}^{k}$ evolve over an interval of length $l \leq\left\lceil\frac{N}{c}\right\rceil-1$, over which $c l$ transmission possibilities exist. It is easy to show that

$$
c l \leq c\left(\left\lceil\frac{N}{c}\right\rceil-1\right)<N
$$

We conclude from (15) that at least one sub-system will not be allowed to transmit over the interval with length $l$, due to capacity limitations, (at worst, $N-c$ sub-systems might not transmit, if the same $c$ sub-systems transmit subsequently at every time-step). Recall that the policy (9) makes scheduling decisions based on both local system parameters, and random noise realizations. Thus, there exist non-zero probabilities for each one of sub-systems to be discarded from transmission. Let sub-system $i$ with arbitrary initial state $\left(x_{0}^{i}, e_{0}^{i}\right)$ be the system that does not certainty transmit over the interval $[0, l]$. Computing $\mathrm{E}\left[\left\|e_{l}^{i}\right\|_{2}^{2}\right]$ at the final time-step of the interval $[0, l]$ results in:

$$
\mathrm{E}\left[\left\|e_{l}^{i}\right\|_{2}^{2}\right]=\left\|A_{i}^{l} e_{0}^{i}\right\|_{2}^{2}+\operatorname{tr}\left(W_{i}\right)+\mathrm{E}\left[\left\|A_{i}^{l-1} w_{0}^{i}+A_{i}^{l-2} w_{1}^{i}+\ldots+A_{i} w_{l-2}^{i}\right\|_{2}^{2}\right] .
$$

Calculating the drift (14) over the interval $[0, l]$ certifies convergence of $\left\{e_{k}\right\}^{k}$ only for certain values of $\left\|A_{i}\right\|, W_{i}$, and $e_{0}^{i}$, which shows conservativeness of the considered analysis tool. To avoid such conservativeness, we define the multi-step drift operator over time intervals with minimum length $\left\lceil\frac{N}{c}\right\rceil$. Showing that the $\delta_{k}$-controlled Markov process $\left\{e_{k}\right\}^{k}$ is convergent (see Theorem 2) ensures that it is an ergodic state. Having ergodic $\left\{e_{k}\right\}^{k}$ together with the existence of the stabilizing controllers $u_{k}^{i}$ guarantee stochastic stability of the overall NCS over the entire state-space.

Therefore, in the followings we investigate $f$-ergodicity of the Markov chain $\left\{e_{k}\right\}^{k}$ under the capacity constraint (10), by investigating the drift operator (14) over any interval of length $\left\lceil\frac{N}{c}\right\rceil=N$, i.e. initiating from time-step $k$, over the interval $[k, k+N]$. It is worth reminding that convergence over an interval implies convergence over longer intervals which include the original interval (refer to (Meyn \& Tweedie, 1996, Ch.19) for discussions on ergodicity over multi-step intervals). 


\section{$3.2 f$-ergodicity of stochastic NCS}

To infer $f$-ergodicity, we employ multi-step drift operator defined in $(14)$, over the interval $[k, k+N]$ :

$$
\Delta V\left(e_{k}, N\right)=\mathrm{E}\left[V\left(e_{k+N}\right) \mid e_{k}\right]-V\left(e_{k}\right), e_{k} \in \mathbb{R}^{n} .
$$

In the followings, we first study stochastic stability of the described NCS assuming ideal communication channel, i.e. no packet is dropped while being transmitted through the channel. Then, we extend the results for the non-ideal case by considering non-zero probability of packet dropouts.

Theorem 2: Consider an NCS consisting of N heterogeneous LTI stochastic sub-systems modeled as (1), and a transmission channel subject to the constraint (10), and the control, estimation and scheduling laws given by (4), (5) and (9), respectively. Assume that all scheduled data packets are successfully transmitted, i.e. $\gamma_{k}^{i}=1$, for all $i \in\{1, \ldots, N\}$ and at all time-steps $k$. Then, the described NCS with the overall network state $\left(x_{k}, e_{k}\right):=\left(x_{k}^{1}, \ldots, x_{k}^{N}, e_{k}^{1}, \ldots, e_{k}^{N}\right)$ is $f$-ergodic.

Proof. We briefly describe the proof idea, while a detailed proof is provided in Appendix A.1. We show that the described NCS with the overall state $\left(x_{k}, e_{k}\right)$ is $f$-ergodic over the entire state space, independent of the initial conditions. Knowing that the overall system state $x_{k}$ is convergent by the stabilizing gains $L_{i}$ 's, we calculate the $N$-step drift (16) considering all possible path transitions of $\left\{e_{k}\right\}^{k} \in \mathbb{R}^{n}$ over the interval $[k, k+N]$. Showing that $\Delta V\left(e_{k}, N\right)$ satisfies the conditions of Theorem 1 , the existence of a compact set, so called $\mathcal{D}_{1}$, is ensured towards which the controlled Markov chain $e_{k}$ is expected to converge with probability one, at least once over every time interval of length $N$. This proves the Markov chain is stationary, and stability then readily follows.

Remark 3: The compact set $\mathcal{D}_{1}$ is an $n$-dimensional set which is composed of $N$ compact sub-sets with dimensions $n_{i}$, where each sub-set is attractive for the corresponding local error state $e_{k}^{i}$. In fact, Theorem 2 shows that if the Markov chain $\left\{e_{k}\right\}^{k}$ evolves outside the compact set $\mathcal{D}_{1} \subset \mathbb{R}^{n}$, it converges inside the set in finite time under the TOD scheduling law (See Appendix A.2.).

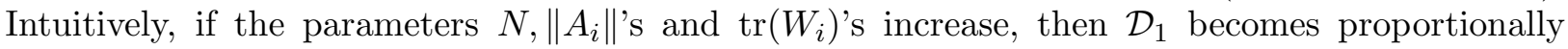
larger in order to satisfy the condition $f \geq 1$ for $e_{k} \notin \mathcal{D}_{1}$, (see Theorem 1). Hence, it concludes that if the channel capacity is fixed, size of the compact set $\mathcal{D}_{1}$ strictly increases by increasing $N$, $\left\|A_{i}\right\|$, and $\operatorname{tr}\left(W_{i}\right)$, which intuitively implies that the Markov chain $\left\{e_{k}\right\}^{k}$ converges to a larger set.

Remark 4: In (Walsh et al., 2002, Theorem 1), global exponential stability (GES) for NCSs consisting of LTI non-disturbed sub-systems under the TOD scheme is shown by the introduction of MATI and placing conservative bounds on the transmission deadline. In the present work, however, such an approach cannot be followed due to the existence of unbounded stochastic disturbances which imply the event-triggers to be stochastic as well. Unlike the results in (Walsh et al., 2002, Lemma 1), the maximum growth of the network-induced error (7) between two consecutive transmissions cannot be uniformly upper-bounded, because random disturbances take arbitrary values. As a result, an upper-bound for the transmission deadline cannot be obtained as a function of system parameters, because transmissions are determined additionally as function of disturbance realizations at each time-step. The notion of $f$-ergodicity in this work is in fact a more general stability criterion than GES, i.e., GES implies $f$-ergodicity. Moreover, unlike (Walsh et al., 2002) which assumes that the network-induced error satisfies a linear growth bound and therefore treats the error as a vanishing perturbation (see (Khalil, 2002), p. 340), f-ergodicity guarantees convergence of $\left\{e_{k}\right\}^{k}$ to a small set with the boundaries defined by the system parameters and noise variance, (See Section 4.). This means that $f$-ergodicity provides stability margins which are not as tight as GES. On the other hand, as it is shown in Theorem 2, f-ergodicity is guaranteed without requiring a transmission deadline, i.e. no necessity of a transmission over every MATI is needed. 


\subsubsection{Stability of Stochastic NCSs Subject to Packet Dropouts}

In this section, we study stochastic stability of NCSs described in (1)-(7) under the TOD scheduling policy (9) considering that every transmitted data packet might be dropped in the communication channel. As already introduced in (3), an acknowledgement signal of the binary form $\gamma_{k}^{i} \in\{0,1\}$ represents that a transmission correspond to sub-system $i$ at time-step $k$ has been successful or not. The dynamics of the error state $e_{k}^{i}$ then depends on both variables $\delta_{k}^{i}$ and $\gamma_{k}^{i}$ as follows:

$$
e_{k+1}^{i}=\left(1-\delta_{k}^{i} \gamma_{k}^{i}\right) A_{i} e_{k}^{i}+w_{k}^{i},
$$

Conventionally, we assume that packet dropouts are modeled by a Bernoulli process. Thus, a transmitted packet is successfully received with probability $p$, or dropped with probability $1-p$, i.e.

$$
\mathrm{P}\left[\gamma_{k}^{i}=1 \mid \delta_{k}^{i}=1\right]=p \quad, \quad \mathrm{P}\left[\gamma_{k}^{i}=0 \mid \delta_{k}^{i}=1\right]=1-p .
$$

Considering the packet loss probability (18), the constraint (10) will be modified as follows:

$$
\sum_{i=1}^{N} \delta_{k}^{i} \gamma_{k}^{i} \leq 1, \quad \forall k \geq 0
$$

In the following theorem, we derive a lower bound on successful transmission probability $p$ such that stochastic stability of the described NCS is guaranteed under the TOD scheduling policy (9).

Theorem 3: Consider an NCS consisting of N heterogeneous LTI stochastic sub-systems modeled as (1) transmitting information over a shared communication channel subject to the constraint (19), and the control, estimation and scheduling laws given by (4), (5) and (9), respectively. Assume that the scheduled data packets are dropped with probability $1-p$, as described in (18). Let

$$
p>1-\frac{1}{\sum_{i=1}^{N}\left\|A_{i}\right\|_{2}^{2 N}}
$$

then the NCS with the overall network state $\left(x_{k}, e_{k}\right):=\left(x_{k}^{1}, \ldots, x_{k}^{N}, e_{k}^{1}, \ldots, e_{k}^{N}\right)$ is f-ergodic.

Proof. The idea of this proof is similar to that of Theorem 2, however the dropout probability is associated in computing the $N$-step drift (16). Since the overall NCS is not stochastically stable for all values of $p \in(0,1)$, we derive the necessary and sufficient stability condition, in form of a lower bound on successful probability $p$. Then, the existence of a compact set, denoted as $\mathcal{D}_{2}$, is ensured toward which the Markov process $\left\{e_{k}\right\}^{k}$ converges. (See Appendix A.2 for the detailed proof.)

Remark 5: The result of Theorem 2 is achievable from Theorem 3 by setting $p=1$. Note that in case $p \in(0,1)$, size of the compact set $\mathcal{D}_{2}$ is expectantly greater than that of the set $\mathcal{D}_{1}$. Intuitively, when a packet is dropped none of the local error states is reset. In expectation, this increases in the overall error norm, and consequently, implies larger convergence set. Moreover, the upper-bound (20) is strictly decreasing with decreasing $\left\|A_{i}\right\|$ and $N$. This observation is expected since dropouts may have greater negative effects on the stability margins if the number of sub-systems increases while the capacity is fixed, or if there exist sub-systems with larger system matrix spectrum.

\section{Performance Analysis}

In this section, we evaluate the overall NCS performance under the TOD scheduling policy (9). By introducing a per-time-step cost function, we derive analytic uniform upper-bounds for the average cost function. We discussed in Section 2 that the control and scheduling laws are independently 
designed. This property allows us to evaluate control performance and scheduling performance also independently. In this article, our attention is focused on evaluating performance of the TOD scheduling policy. Therefore, we define the per-time-step cost function $J_{e_{k}}: \mathbb{R}^{n} \rightarrow \mathbb{R}_{\geq 0}$, as follows:

$$
J_{e_{k}} \triangleq \frac{1}{N} \sum_{i=1}^{N} e_{k}^{i^{\top}} e_{k}^{i}=\frac{1}{N} \sum_{i=1}^{N}\left\|e_{k}^{i}\right\|_{2}^{2}
$$

According to (21), there is no penalty on the control-related signals $u_{k}^{i}$ and $x_{k}^{i}$. However, due to the independence of the control inputs from the scheduling policy, terms involving $u_{k}^{i}$ and $x_{k}^{i}$ can readily be added to the cost (21). A potential minimization of such terms would directly affect the design of control gain $L_{i}$, (Molin \& Hirche, 2011). This is however out of scope of this article.

From the per-time-step cost function (21), we define the average cost function $J_{\text {ave }}$ as

$$
J_{\text {ave }}=\lim _{T \rightarrow \infty} \sup \frac{1}{T} \sum_{k=0}^{T-1} \mathrm{E}\left[J_{e_{k}}\right] .
$$

For the purpose of brevity, we only obtain performance bounds for the case without packet dropout, i.e. $p=1$. The results are readily extendable for the scenario with packet loss, i.e. $0<p<1$.

Theorem 4: Consider an NCS described in (1)-(7), with the individual control loops exchanging data over a shared communication channel subject to the constraint (10). Assume that the data packets are scheduled for transmission according to the TOD policy given in (9) and assume $p=1$ in (18). Then the average cost (22) is uniformly bounded by the following upper-bound:

$$
J_{\text {ave }} \leq \sum_{i=1}^{N} \sum_{r=0}^{N-1} \operatorname{tr}\left(W_{i}\right)\left\|A_{i}\right\|_{2}^{2(N-r-1)}
$$

Proof. See Appendix A.3.

The uniform upper-bound (23) is an strictly increasing function w.r.t. increasing $\left\|A_{i}\right\|, \operatorname{tr}\left(W_{i}\right)$, and $N$ for all $i \in\{1, \ldots, N\}$. This is expected since the per-time-step cost function $J_{e_{k}}$ is dependent on the quadratic form of the error, which for a fixed capacity increases by increasing $\left\|A_{i}\right\|, \operatorname{tr}\left(W_{i}\right)$, and $N$. Moreover, (23) does not expose the dependency on channel capacity $c$, since the capacity $c$ is assumed to be 1, according to (10). Expectantly, considering $c>1$ provides the possibility of having more than one transmission at each time-step, leading to a tighter performance bound.

Corollary 1: Consider the NCS setup described in Theorem 2. Let $\mathcal{D} \subset \mathbb{R}^{n}, n=\sum_{i=1}^{N} n_{i}$, be a given measurable subset with the Lebesgue measure $\lambda(\mathcal{D})$, such that for a non-negative real-valued function $V\left(e_{k}\right)$, and $f\left(e_{k}\right) \geq 1, \Delta V\left(e_{k}, N\right) \leq-f$ for $e_{k} \notin \mathcal{D}$, and $\Delta V\left(e_{k}, N\right)<\infty$, for $e_{k} \in \mathcal{D}$. If

$$
\lambda(\mathcal{D}) \geq \sum_{i=1}^{N} \operatorname{tr}\left(W_{i}\right)^{\frac{1}{2}}\left(\sum_{r=0}^{N-1}\left\|A_{i}\right\|_{2}^{2(N-r-1)}\right)^{\frac{1}{2}},
$$

then, $\left\{e_{k}\right\}^{k} \stackrel{\text { exp }}{\longrightarrow} \mathcal{D}$ as $k \rightarrow \infty$, for all $e_{k} \notin \mathcal{D}$, where, $\stackrel{\text { exp }}{\longrightarrow}$ denotes the convergence in expectation.

Proof. According to the Theorem 2, the Markov process $\left\{e_{k}\right\}^{k}$ is $f$-ergodic, and hence a stationary process, under the TOD scheduling law. Moreover, according to the Theorem 4, we can derive $\mathrm{E}\left[\left\|e_{k+N}\right\|_{2}\right]=\sum_{i=1}^{N} \mathrm{E}\left[\left\|e_{k+N}^{i}\right\|_{2}\right] \leq \sum_{i=1}^{N} \operatorname{tr}\left(W_{i}\right)^{\frac{1}{2}}\left(\sum_{r=0}^{N-1}\left\|A_{i}\right\|_{2}^{2(N-r-1)}\right)^{\frac{1}{2}}$. This guarantees that $\left\{e_{k}\right\}^{k}$ converges in expectation to a Lebesgue measurable compact set $\mathcal{D}$, with probability one, at most every $N$ time-steps, if the Lebesgue measure ${ }^{3} \lambda(\mathcal{D})$ satisfies the inequality (24). It guarantees asymptotic convergence of $\left\{e_{k}\right\}^{k}$ to $\mathcal{D}$ in expectation, and the proof then readily follows.

${ }^{3}$ Remind that for every Borel measurable set, the Borel and the Lebesgue measures coincide. 
Table 1. NCS simulations parameters.

\begin{tabular}{lcccccc}
\hline & $A_{i}$ & $B_{i}$ & $L_{i}=A_{i} B_{i}^{-1}$ & $x_{0}^{i}$ & $w_{k}^{i}$ \\
\hline Class $c l_{1}$ with $N_{u}{ }^{*}$ unstable sub-systems & 1.25 & 1 & 1.25 & 0 & i.i.d. with $\mathcal{N}\left(0, W_{i}\right)$ \\
Class $c l_{2}$ with $N_{s}{ }^{*}$ stable sub-systems & 0.75 & 1 & 0.75 & 0 & i.i.d. with $\mathcal{N}\left(0, W_{i}\right)$ \\
\hline
\end{tabular}

* Total number of sub-systems in an NCS equals $N$, where $N=N_{s}+N_{u}$.

From corollary 1 one concludes that in the presence of stochastic system disturbances, the Markov process $\left\{e_{k}\right\}^{k}$ does not generally converge to zero, but to a compact set with the boundaries dependent on $\operatorname{tr}\left(W_{i}\right),\left\|A_{i}\right\|$, and $N$. However, the result of (Walsh et al., 2002), Lemma 1, can be deduced from the expression (24). In fact, in the absence of stochastic disturbances, the error asymptotically converges to the origin with zero Lebesgue measure, i.e. the error is a vanishing perturbation. In addition, from the corollary 1 , we can determine the system parameters $\operatorname{tr}\left(W_{i}\right)$, $\left\|A_{i}\right\|$, and $N$ in order to achieve a desired performance. Additionally, for given parameters, we can determine the minimal compact set towards which the error is converging. It is worth reminding that the provided lower-bound (24) is only a sufficient condition and is moderately conservative.

\section{Simulation Results}

In this section we validate the theoretical claims made in this article through simulating multi-loop NCSs with different parameter settings. To highlight the efficacy of the event-triggered channel arbitration, we compare the performance of the TOD scheduler with some of the conventional policies such as time-triggered TDMA, static random MAC, and event-based uniform access arbitration.

To account for the system heterogeneity, each sub-system in an NCS belongs to one of the two classes of sub-systems, namely $\left\{c l_{1}, c l_{2}\right\}$, where in one class the systems are homogeneous. The class $c l_{1}$ includes unstable sub-systems, and the class $c l_{2}$ contains stable sub-systems. For the sake of illustrations, we consider scalar sub-systems in the simulations, with the parameters summarized in Table 1 for each class. Each individual sub-system $i$ is steered by a dead-beat control law with stabilizing gain $L_{i}=A_{i} B_{i}^{-1}$. Recalling the closed-loop dynamics (6), the dead-beat control incurs the dynamics to be solely error dependent, i.e. $x_{k+1}^{i}=\left(1-\theta_{k}^{i}\right) A_{i} e_{k}^{i}+w_{k}^{i}$, which exactly resembles the error dynamics in (7). Therefore, it is sufficient to simulate the dynamic behavior of the error state. We conduct Monte Carlo simulations for each NCS setup with $10^{5}$ samples, and confidence intervals are computed over 10 runs. As $\left\{e_{k}\right\}^{k}$ is a random sequence with Gaussian-distributed random components $e_{k}^{i}$, we look at the variance of the distribution of $e_{k}$. To demonstrate the numerical results comprehensively, we conduct the simulations for different sets of system-related and channel-related parameters. In below, we provide comparisons for the two discussed scenarios: 1) ideal communication channels, and 2) error-prone channels with possibility of packet loss.

\subsection{Ideal communication}

Consider an NCS consisting of 100 scalar LTI sub-systems with 50 sub-systems belonging to each class $c l_{1}$ and $c l_{2}$, i.e. $N_{u}=50$ and $N_{s}=50$. Let $p=1$, i.e. scheduled data packets are not dropped out. The simulative aggregate error variances versus different ratios $\frac{c}{N}=\{0.05,0.1,0.2,0.3,0.4,0.5\}$, (i.e. for varying number of parallel transmission slots $c=\{5,10,20,30,40,50\})$ are depicted in Fig. 2 (a). Additionally, the variances are plotted in Fig. 2 (a) for Gaussian disturbances with various second moments $W=\{0.5,1,2,3,4\}$. As expected, the aggregate variances decrease by increasing the channel capacity $c$. In addition, the more uncertain the process disturbance is, i.e. greater $W$, the higher the aggregate error variance becomes. In case the channel capacity is quite scarce, e.g. $\frac{c}{N}=0.05$ in Fig. 2 (a), the aggregate error variance becomes largely sensitive to 
the process disturbance. This observation can be explained by noting that the local error states of unstable sub-systems grow rapidly if they do not transmit often enough, which occurs when resource scarcity increases. Since local errors are marred by random disturbances, more uncertain disturbance distributions yields even faster growth rate for local errors. This observation is also in accordance with the theoretic results of Theorem 2 (see the expression (A6) in the Appendix A.1.).

In Fig. 2 (b), the comparisons are made between scenarios with different number of unstable sub-systems $N_{u}=\{60,70,80,90,100\}$, in an NCS with $N=100$, while the variance of the process noise is set to be fixed at $W=1$. The aggregate error variances are plotted versus the ratios $\frac{c}{N}=\{0.1,0.2,0.3,0.4,0.5\}$. As it can be seen, the aggregate error variances conceivably increase with increasing $N_{u}$. However, the gap is more evident when the channel capacity is quite limited, see e.g. for $\frac{c}{N}=0.1$. For higher transmission possibilities though, the gap decays drastically, see e.g. the negligible gap between different curves for $\frac{c}{N}=0.5$. The reason is, waiting time for a transmission is expectedly shorter for unstable sub-systems when $\frac{c}{N}$ increases, which prevents the corresponding errors to be increased exponentially. In fact, Fig. 2 (b) illustrates that the event-based scheduling is hugely beneficial for stochastic NCSs in case communication resources are moderately scarce. This can be seen by comparing the error variances for two cases $N_{u}=60$ and $N_{u}=100$, when $\frac{c}{N}=0.5$.

\subsection{Binary erasure channel - Packet loss}

Next, we evaluate the effects of packet loss on the overall NCS performance. In Fig. 3 (a), the aggregate error variances are shown versus different ratios of $\frac{c}{N}=\{0.1,0.2,0.3,0.4,0.5\}$ for various dropout probabilities. The total number of sub-systems equals 100 and $W=1$. A fixed success probability $p$ is assigned to each sub-system and $\gamma_{k}^{i}$ is determined as the outcome of the Bernoulli distribution such that $\gamma_{k}^{i}=1$ w.p. $p$, and $\gamma_{k}^{i}=0$ w.p. $1-p$. As it is illustrated in Fig. 3 (a), the error variances increase with decreasing $p$. However, the gap becomes narrower as $\frac{c}{N}$ increases.

Fig. 3 (b) provides performance comparisons between the TOD scheduling scheme and TDMA, static uniform MAC, and the event-triggered threshold policy, for NCSs with different number of sub-systems $N \in\{2,4,6,8,10,20\}$, subject to the capacity constraint (19). Each stable and unstable class include $\frac{N}{2}$ sub-systems. To have a fair performance comparison between TOD and TDMA, we derived optimal transmission pattern for the latter periodic scheme by brute force search over a finite time window. Remind that finding the optimal TDMA pattern in infinite horizon is NP-hard. Thus, we numerically search for TDMA pattern which results in the minimum error variance over

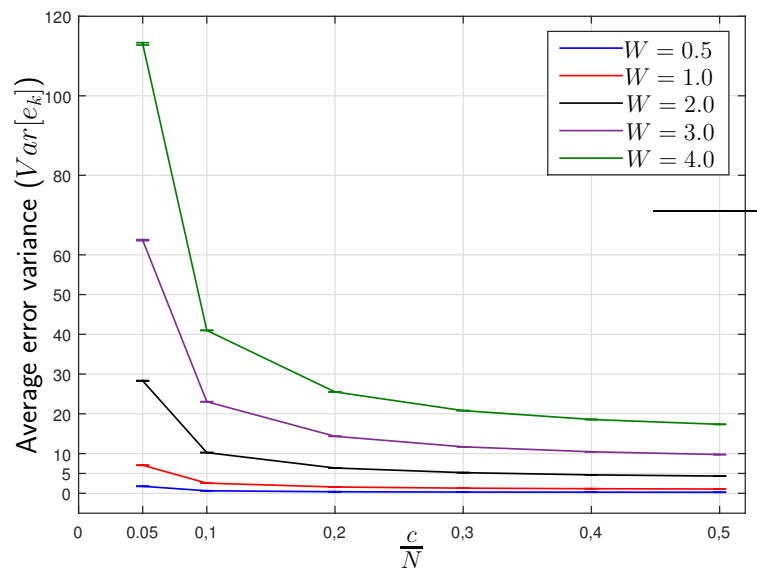

(a) Average error variance vs. $\frac{c}{N}$ for different variance values of process noise $W=\{0.5,1.0,2.0,3.0,4.0\}$.

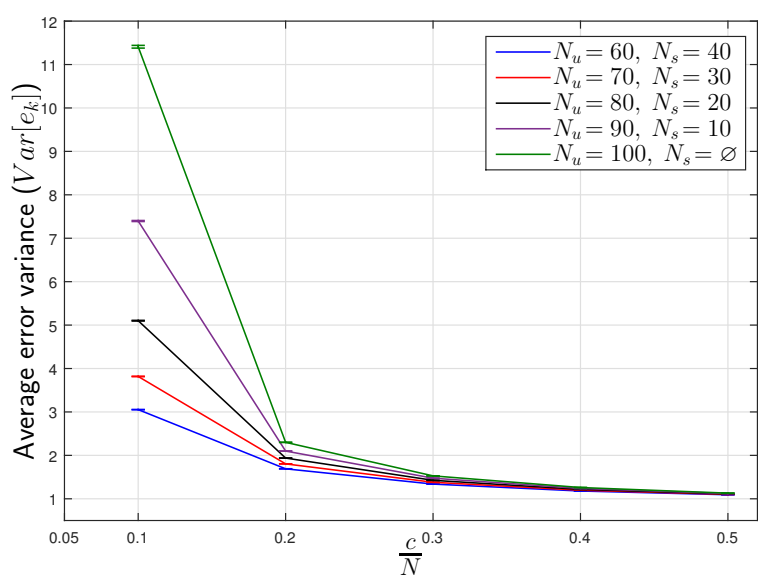

(b) Average error variance vs. $\frac{c}{N}$ for different number of unstable sub-systems $N_{u}=\{60,70,80,90,100\}$.

Figure 2. Aggregate mean error variance vs. $\frac{c}{N}$ for $N=100$. 


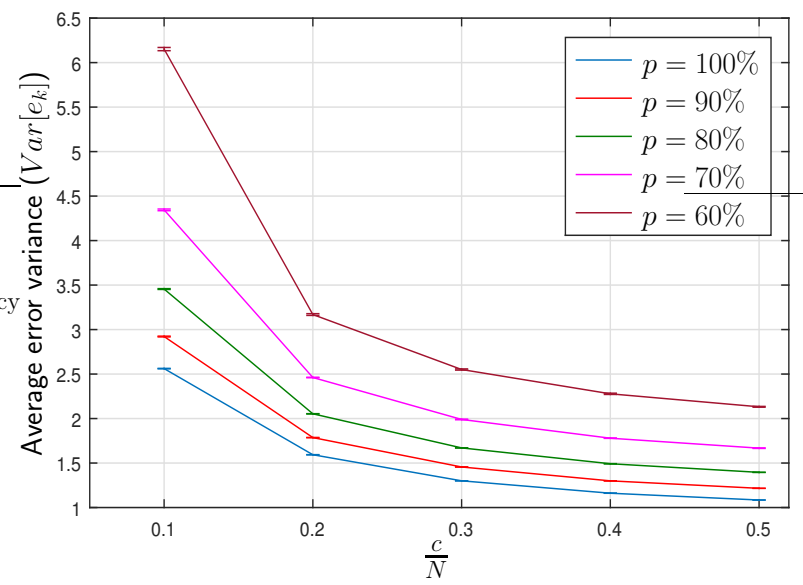

(a) Average error variance vs. $\frac{c}{N}$ for different packet dropout probabilities $1-p=\{0,0.05,0.1,0.15,0.2,0.3,0.4\}$.

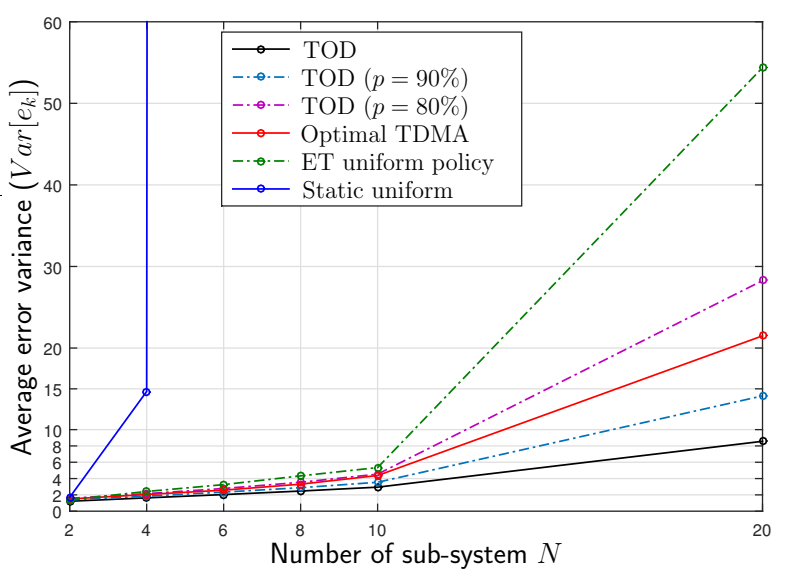

(b) Average error variance vs. number of sub-systems $N=$ $\{2,4,6,8,10,20\}$ with $W=1.0$, for different MAC protocols.

Figure 3. Comparison of aggregate mean error variance.

a finite time window for each set-up with $N \in\{2,4,6,8,10,20\}$. This search is however utterly exhaustive for more sub-systems and over longer time windows (The search for $N=4$ over 9 timesteps lasts nearly 11 hours on a $3.90 \mathrm{GHz} 4690$ Core i5 CPU). Moreover, the optimal pattern changes sensitively w.r.t. the system parameters, hence not an appropriate design method for large scale NCSs. We also compare performance of the TOD approach with static scheduling which is a non-prioritized channel arbitration with pre-given transmission chance of $\frac{A_{i}^{2}}{\sum_{j=1}^{N} A_{j}^{2}}$ for a sub-system $i$ at every time-step. (This is an improvement to the standard CSMA with access probability $\frac{1}{N}$ ). Fig. 3 (b), shows the static protocol results in acceptable variance only up to $N=4$, while for $N=6$ it takes the value with magnitude $8 \times 10^{3}$. This is an expected observation due to the static nature of resource allocation which may result in long non-transmission windows for unstable systems.

We additionally compare the performance of TOD with the event-triggered threshold-based scheduling approach, proposed in Molin and Hirche (2014a) (green dotted curve in Fig. 3 (b)). According to this approach, a sub-system is qualified for transmission if a function of its state exceeds some pre-given thresholds, otherwise it is excluded. In case the number of qualified subsystems is greater than the channel capacity $c$, the scheduler randomly selects $c$ sub-systems of the qualified ones uniformly while the rest are expelled. Fig. 3 (b) shows that, for $p=1$, TOD approach outperforms all mentioned schemes, especially when the size of the NCS increases. Since packet dropout is not considered for the other protocols, it is fair to compare them with TOD only for $p=1$. The two blue and purple dotted curves represent the performance of TOD with successful transmission probabilities $90 \%$ and $80 \%$, respectively. It concludes that the theoretical lower bound for the success probability $p$ in (20) is excessively conservative. According to (20), for the given NCS parameters, success probability to guarantee stability can be computed for $N=\{2,4,6,8,10,20\}$ which results in $p>\{0.6374,0.9175,0.9771,0.9930,0.9977,0.9999\}$, respectively. However, Fig. 3 (b) shows, e.g. for $p=0.8$ and $N=20$, the aggregate error variance is still well-bounded.

\section{Conclusion}

In this article, we employ the event-based TOD scheduling scheme as the governing unit to allocate the scarce communication resources among multiple stochastic LTI sub-systems in an NCS. We then address stochastic stability and analyze performance of the described networked system considering that the communication channel is subject to probabilistic transmission failure in form of random data packet loss. Showing that the resulting overall network state has Markovian property, stability 
properties of such networked systems under the TOD scheduling scheme is investigated within the stochastic concept of $f$-ergodicity. In the presence of Bernoulli-distributed random packet dropouts, sufficient stability conditions are derived to guarantee the overall NCS stability. Furthermore, a uniform analytic performance bound for an average social cost function is derived under the employment of the TOD rule. Comprehensive numerical analysis validate our stability claim and show boundedness of the aggregate error variance with and without possibility of packet loss. In addition, we show a major performance improvement, in case the TOD approach is employed, in comparison with related time-triggered, event-triggered, and randomized scheduling policies.

\section{Acknowledgement}

This work is funded by the German Research Foundation (DFG) within the Priority Program SPP 1914 "Cyber-Physical Networking".

\section{References}

Al-Areqi, S., Grges, D., \& Liu, S. (2015). Event-based control and scheduling codesign: Stochastic and robust approaches. IEEE Transactions on Automatic Control, 60(5), 1291-1303.

Antunes, D., Heemels, W., \& Tabuada, P. (2012). Dynamic programming formulation of periodic event-triggered control: Performance guarantees and co-design. In Decision and control, IEEE 51st annual conference on (p. 7212-7217).

Åström, K., \& Bernhardsson, B. (2002). Comparison of Riemann and Lebesgue sampling for first order stochastic systems. In 41st IEEE conference on decision and control (p. 2011-2016).

Bauer, N., Donkers, M., van de Wouw, N., \& Heemels, W. (2013). Decentralized observer-based control via networked communication. Automatica, 49(7), 2074 - 2086.

Blind, R., \& Allgöwer, F. (2011a). Analysis of networked event-based control with a shared communication medium: Part II - slotted ALOHA. In 18th IFAC world congress (p. 8830 $-8835)$.

Blind, R., \& Allgöwer, F. (2011b). Analysis of networked event-based control with a shared communication medium: Part I pure ALOHA. IFAC Proceedings, 44(1), $10092-10097$.

Carnevale, D., Teel, A. R., \& Nesic, D. (2007). A lyapunov proof of an improved maximum allowable transfer interval for networked control systems. IEEE Transactions on Automatic Control, 52(5), 892-897.

Cervin, A., \& Henningsson, T. (2008). Scheduling of event-triggered controllers on a shared network. In 47th IEEE conference on decision and control (p. 3601-3606).

Christmann, D., Gotzhein, R., Siegmund, S., \& Wirth, F. (2014). Realization of TOD in wireless multihop networks. IEEE Trans. on Industrial Informatics, 10(1), 17-26.

Cogill, R., \& Lall, S. (2006). Suboptimality bounds in stochastic control: A queueing example. In American control conference, 2006 (p. 1642-1647).

Dimarogonas, D., \& Johansson, K. (2009). Event-triggered control for multi-agent systems. In 48th IEEE conf. on decision and control held jointly with 28th Chinese control conf. (p. 7131$7136)$.

Donkers, M., Heemels, W., Bernardini, D., Bemporad, A., \& Shneer, V. (2012). Stability analysis of stochastic networked control systems. Automatica, 48(5), 917-925.

Donkers, M., Heemels, W., van de Wouw, N., \& Hetel, L. (2011). Stability analysis of networked control systems using a switched linear systems approach. IEEE Transactions on Automatic Control, 56(9), 2101-2115.

Duc, L. H., Christmann, D., Gotzhein, R., Siegmund, S., \& Wirth, F. (2015). The stability of tryonce-discard for stochastic communication channels: Theory and validation. In 54th IEEE conference on decision and control (cdc) (p. 4170-4175).

Feller, W. (1971). An introduction to probability theory and its applications (Vol. 2). John Wiley \& Sons Inc. 
Gatsis, K., Ribeiro, A., \& Pappas, G. J. (2015). 5th ifac workshop on distributed estimation and control in networked systems. IFAC-PapersOnLine, 48(22), 209 - 214.

Gupta, R., \& Chow, M.-Y. (2010). Networked control system: Overview and research trends. Industrial Electronics, IEEE Transactions on, 57(7), 2527-2535.

Gupta, V., Dana, A., Hespanha, J., Murray, R., \& Hassibi, B. (2009). Data transmission over networks for estimation and control. Automatic Control, IEEE Trans. on, 54(8), 1807 1819.

Heemels, W., Sandee, J. H., \& Van Den Bosch, P. (2008). Analysis of event-driven controllers for linear systems. International Journal of Control, 81(4), 571-590.

Henningsson, T., Johannesson, E., \& Cervin, A. (2008). Sporadic event-based control of first-order linear stochastic systems. Automatica, 44 (11), 2890 - 2895.

Hernandez Lerma, O., \& Lasserre, J. (2001). Further criteria for positive Harris recurrence of Markov chains. Proceedings of the American Mathematical Society, 129(5), 1521-1524.

Hespanha, J., Naghshtabrizi, P., \& Xu, Y. (2007). A survey of recent results in networked control systems. Proceedings of the IEEE, 95(1), 138-162.

Khalil, H. (2002). Nonlinear systems. Prentice Hall.

Liu, Q., Wang, Z., He, X., \& Zhou, D. (2014). A survey of event-based strategies on control and estimation. Systems Science $\&$ Control Engineering, 2(1), 90-97.

Lunze, J., \& Lehmann, D. (2010). A state-feedback approach to event-based control. Automatica, 46(1), 211-215.

Mamduhi, M., Kneissl, M., \& Hirche, S. (2016). Decentralized event-triggered medium access control for networked control systems. In 55th IEEE conference on decision and control (p. 513-519).

Mamduhi, M., Molin, A., \& Hirche, S. (2014). Event-based scheduling of multi-loop stochastic systems over shared communication channels. In 21st international symposium on mathematical theory of networks and systems (pp. 266-273).

Mamduhi, M., Molin, A., Tolic, D., \& Hirche, S. (2017). Error-dependent data scheduling in resource-aware multi-loop networked control systems. Automatica, 81, 209-216.

Mamduhi, M., Tolic, D., \& Hirche, S. (2015a). Decentralized event-based scheduling for sharedresource networked control systems. In 14th european control conference (pp. 941-947).

Mamduhi, M., Tolic, D., \& Hirche, S. (2015b). Robust event-based data scheduling for resource constrained networked control systems. In American control conf. (p. 4695-4701).

Mamduhi, M., Tolic, D., Molin, A., \& Hirche, S. (2014). Event-triggered scheduling for stochastic multi-loop networked control systems with packet dropouts. In IEEE 53rd annual conf. on decision and control (cdc) (p. 2776-2782).

McKernan, A. D., \& Irwin, G. W. (2010). Event-based sampling for wireless network control systems with QoS. In American control conference (p. 1841-1846).

Meyn, S., \& Tweedie, R. (1996). Markov chains and stochastic stability. Springer London.

Molin, A., \& Hirche, S. (2011). Optimal design of decentralized event-triggered controllers for largescale systems with contention-based communication. In 50th IEEE conference on decision and control and european control conference (p. 4710-4716).

Molin, A., \& Hirche, S. (2013). On the optimality of certainty equivalence for event-triggered control systems. IEEE Transactions on Automatic Control, 58(2), $470-474$.

Molin, A., \& Hirche, S. (2014a). A bi-level approach for the design of event-triggered control systems over a shared network. Discrete Event Dynamic Systems, 24(2), 153-171.

Molin, A., \& Hirche, S. (2014b). Price-based adaptive scheduling in multi-loop control systems with resource constraints. IEEE Trans. on Automatic Control, 59(12), 3282-3295.

Murray, R., Åström, K., Boyd, S., Brockett, R., \& Stein, G. (2003). Future directions in control in an information-rich world. IEEE Control Systems Magazine, 23(2), 20-33.

Nesic, D., \& Teel, A. (2004). Input-output stability properties of networked control systems. IEEE Trans. on Automatic Control, 49(10), 1650-1667. 
Nesic, D., \& Liberzon, D. (2009). A unified framework for design and analysis of networked and quantized control systems. Automatic Control, IEEE Transactions on, 54(4), 732-747.

Rabi, M., Moustakides, G. V., \& Baras, J. S. (2012). Adaptive sampling for linear state estimation. SIAM Journal on Control and Optimization, 50(2), 672-702.

Ramesh, C., Sandberg, H., \& Johansson, K. (2013). Design of state-based schedulers for a network of control loops. Automatic Control, IEEE Transactions on, 58(8), 1962-1975.

Shi, L., Epstein, M., Sinopoli, B., \& Murray, R. M. (2007). Effective sensor scheduling schemes in a sensor network by employing feedback in the communication loop. In 2007 IEEE international conference on control applications (pp. 1006-1011).

Tabbara, M., \& Nesic, D. (2008). Input-output stability of networked control systems with stochastic protocols and channels. IEEE Trans. on Automatic Control, 53(5), 1160-1175.

Tabbara, M., Nesic, D., \& Teel, A. (2007). Stability of wireless and wireline networked control systems. Automatic Control, IEEE Transactions on, 52(9), 1615-1630.

Tabuada, P. (2007). Event-triggered real-time scheduling of stabilizing control tasks. Automatic Control, IEEE Transactions on, 52(9), $1680-1685$.

Vilgelm, M., Mamduhi, M., Kellerer, W., \& Hirche, S. (2016). Adaptive decentralized mac for eventtriggered networked control systems. In 19th international conference on hybrid systems: Computation and control (hscc) (pp. 165-4174). Vienna, Austria.

Walsh, G. C., Ye, H., \& Bushnell, L. G. (2002). Stability analysis of networked control systems. IEEE Trans. on Control Systems Technology, 10(3), 438-446.

Wang, X., \& Lemmon, M. (2011). Event-triggering in distributed networked control systems. IEEE Trans. on Automatic Control, 56(3), 586-601.

Zhang, L., Gao, H., \& Kaynak, O. (2013). Network-induced constraints in networked control systems-a survey. Industrial Informatics, IEEE Transactions on, 9(1), 403-416.

\section{Appendix A. Technical Proofs}

\section{A.1 Proof of Theorem 2}

Proof. Remind that existence of the stabilizing control gains $L_{i}$ 's ensure that $x_{k}=\left(x_{k}^{1}, \ldots, x_{k}^{N}\right)$ is mean square stable, and consequently $f$-ergodic, if $\theta_{k}^{i}=1$ for all $i \in\{1, \ldots, N\}$ and $k \in\{0,1, \ldots\}$. In the presence of the constraint (10), $f$-ergodicity of the error process $\left\{e_{k}\right\}^{k}$ ensures the convergence of the overall network state $\left(x_{k}, e_{k}\right)$. As discussed, we analyze convergence of $\left\{e_{k}\right\}^{k}$ over an interval with length $N$ under the TOD policy (9). First, we define two complementary and disjoint sets $G$ and $\bar{G}$, such that set $G$ contains the sub-systems which have transmitted at least once over $[k, k+N]$, and set $\bar{G}$ includes those with no transmission over the same interval. Clearly, $|G|+|\bar{G}|=N$, where $|\cdot|$ denotes the cardinality operator. Since $p=1$, exactly one successful transmission occurs at each time-step implying that $|G| \geq 1$. The $N$-step drift (16) can accordingly be separated as follows:

$$
\Delta V\left(e_{k}, N\right)=\sum_{i \in G} \mathrm{E}\left[\left\|e_{k+N}^{i}\right\|_{2}^{2} \mid e_{k}\right]+\sum_{j \in \bar{G}} \mathrm{E}\left[\left\|e_{k+N}^{j}\right\|_{2}^{2} \mid e_{k}\right]-V\left(e_{k}\right) .
$$

For notation convention, let $i$ and $j$ be the indexes of sub-systems belonging to the sets $G$ and $\bar{G}$, respectively. Recalling $\gamma_{\bar{k}}^{i}=1$, for all $\bar{k} \geq 0, e_{k+N}^{i}$ can be correlated to a previous error $e_{k+r_{i}^{\prime}}^{i}$, as

$$
e_{k+N}^{i}=\prod_{d=r_{i}^{\prime}}^{N-1}\left(1-\delta_{k+d}^{i}\right) A_{i}^{N-r_{i}^{\prime}} e_{k+r_{i}^{\prime}}^{i}+\sum_{r=r_{i}^{\prime}}^{N-1}\left[\prod_{d=r+1}^{N-1}\left(1-\delta_{k+d}^{i}\right) A_{i}^{N-r-1} w_{k+r}^{i}\right]
$$

where, $r_{i}^{\prime} \in[0, N-1], d$ is the scheduling variable counter, and we define $\prod_{d=N}^{N-1}(\cdot) \triangleq 1$.

Let time-step $k+r_{i}$ denote the latest time that the estimation error of sub-system $i \in G$ is reset over the interval $[k, k+N]$, where $r_{i} \in[1, N]$. This means $\delta_{k+r_{i}-1}^{i}=1$ and hence $e_{k+r_{i}}^{i}=w_{k+r_{i}-1}^{i}$, 
according to (7). Therefore, $\delta_{k+\bar{r}_{i}}^{i}=0$, for all $\bar{r}_{i} \in\left[r_{i}, N-1\right]$. We can then express $e_{k+N}^{i}$ as follows

$$
\begin{aligned}
e_{k+N}^{i} & =A_{i}^{N-r_{i}} e_{k+r_{i}}^{i}+\sum_{r=r_{i}}^{N-1} A_{i}^{N-r-1} w_{k+r}^{i} \\
& =A_{i}^{N-r_{i}} w_{k+r_{i}-1}^{i}+\sum_{r=r_{i}}^{N-1} A_{i}^{N-r-1} w_{k+r}^{i}=\sum_{r=r_{i}-1}^{N-1} A_{i}^{N-r-1} w_{k+r}^{i} .
\end{aligned}
$$

As stochastic disturbance process is i.i.d at every time-step, employing (A3) for all $i \in G$ yields

$$
\begin{aligned}
\sum_{i \in G} \mathrm{E}\left[\left\|e_{k+N}^{i}\right\|_{2}^{2} \mid e_{k}\right] & =\sum_{i \in G} \mathrm{E}\left[\sum_{r=r_{i}-1}^{N-1}\left\|A_{i}^{N-r-1} w_{k+r}^{i}\right\|_{2}^{2}\right] \\
& \leq \sum_{i \in G} \sum_{r=r_{i}-1}^{N-1} \operatorname{tr}\left(W_{i}\right)\left\|A_{i}^{N-r-1}\right\|_{2}^{2}
\end{aligned}
$$

Now, assume $|\bar{G}|=m$, where $m \in[0, N-1]$. If $m=0$, then all $N$ sub-systems transmit over the interval $[k, k+N]$ each exactly once, and (A4) is valid for all sub-systems. In case $m>0$, then there exists at least one sub-system, say $q \in G$, which has transmitted more than once over $[k, k+N]$. Assume $k+r_{q}$ and $k+r_{q q}$ are the two latest reset times of sub-system $q$, where $r_{q}<r_{q q} \leq N$, i.e. $\delta_{k+r_{q}-1}^{q}=1$ and $\delta_{k+r_{q q}-1}^{q}=1$. This implies that $e_{k+r_{q}}^{q}=w_{k+r_{q}-1}^{q}$. From (A2), the following holds

$$
\begin{aligned}
e_{k+r_{q q}-1}^{q} & =\prod_{d=r_{q}}^{r_{q q}-2}\left(1-\delta_{k+d}^{q}\right) A_{q}^{r_{q q}-r_{q}-1} e_{k+r_{q}}^{q}+\sum_{r=r_{q}}^{r_{q q}-2}\left[\prod_{d=r+1}^{r_{q q}-2}\left(1-\delta_{k+d}^{q}\right) A_{q}^{r_{q q}-r-2} w_{k+r}^{q}\right] \\
& =A_{q}^{r_{q q}-r_{q}-1} w_{k+r_{q}-1}^{q}+\sum_{r=r_{q}}^{r_{q q}-2} A_{q}^{r_{q q}-r-2} w_{k+r}^{q}=\sum_{r=r_{q}-1}^{r_{q q}-2} A_{q}^{r_{q q}-r-2} w_{k+r}^{q} .
\end{aligned}
$$

As $\delta_{k+r_{q q}-1}^{q}=1$, for all $j \in \bar{G}$ we have $\left\|e_{k+r_{q q}-1}^{q}\right\|_{2}>\left\|e_{k+r_{q q}-1}^{j}\right\|_{2}$. We also have from (A2) for a $j \in \bar{G}$

$$
e_{k+N}^{j}=A_{j}^{N-r_{q q}+1} e_{k+r_{q q}-1}^{j}+\sum_{r=r_{q q}-1}^{N-1} A_{j}^{N-r-1} w_{k+r}^{j}
$$

Since sub-systems $j \in \bar{G}$ never transmit, $\delta_{\bar{k}}^{j}=0$ for all $\bar{k} \in[k, k+N]$. Therefore, we simply obtain

$$
\begin{aligned}
\mathrm{E}\left[\left\|e_{k+N}^{j}\right\|_{2}^{2} \mid e_{k}\right] & =\mathrm{E}\left[\left\|A_{j}^{N-r_{q q}+1} e_{k+r_{q q}-1}^{j}+\sum_{r=r_{q q}-1}^{N-1} A_{j}^{N-r-1} w_{k+r}^{j}\right\|_{2}^{2} \mid e_{k}\right] \\
& \leq\left\|A_{j}^{N-r_{q q}+1}\right\|_{2}^{2} \mathrm{E}\left[\left\|e_{k+r_{q q}-1}^{q}\right\|_{2}^{2} \mid e_{k}\right]+\sum_{r=r_{q q}-1}^{N-1} \operatorname{tr}\left(W_{j}\right)\left\|A_{j}^{N-r-1}\right\|_{2}^{2} \\
& \leq\left\|A_{j}^{N-r_{q q}+1}\right\|_{2}^{2} \sum_{r=r_{q}-1}^{r_{q q}-2} \operatorname{tr}\left(W_{q}\right)\left\|A_{q}^{r_{q q}-r-2}\right\|_{2}^{2}+\sum_{r=r_{q q}-1}^{N-1} \operatorname{tr}\left(W_{j}\right)\left\|A_{j}^{N-r-1}\right\|_{2}^{2} .
\end{aligned}
$$

Having (A4) and (A7), the $N$-step drift operator (A1) reduces to

$$
\begin{aligned}
\Delta V\left(e_{k}, N\right) & \leq \sum_{i \in G} \sum_{r=r_{i}-1}^{N-1} \operatorname{tr}\left(W_{i}\right)\left\|A_{i}^{N-r-1}\right\|_{2}^{2}+\sum_{j \in \bar{G}} \sum_{r=r_{q q}-1}^{N-1} \operatorname{tr}\left(W_{j}\right)\left\|A_{j}^{N-r-1}\right\|_{2}^{2} \\
& +\sum_{j \in \bar{G}}\left\|A_{j}^{N-r_{q q}+1}\right\|_{2}^{2} \sum_{r=r_{q}-1}^{r_{q q}-2} \operatorname{tr}\left(W_{q}\right)\left\|A_{q}^{r_{q q}-r-2}\right\|_{2}^{2}-V\left(e_{k}\right) .
\end{aligned}
$$

Define the real-valued function $f_{1}=\epsilon_{1} V\left(e_{k}\right)-\xi$, with $\epsilon_{1} \in(0,1]$ and bounded variable $\xi>0$ as

$$
\xi=\sum_{i \in G} \sum_{r=r_{i}-1}^{N-1} \operatorname{tr}\left(W_{i}\right)\left\|A_{i}^{N-r-1}\right\|_{2}^{2}+\sum_{j \in \bar{G}}\left[\left\|A_{j}^{N-r_{q q}+1}\right\|_{2}^{2} \sum_{r=r_{q}-1}^{r_{q q}-2} \operatorname{tr}\left(W_{q}\right)\left\|A_{q}^{r_{q q}-r-2}\right\|_{2}^{2}+\sum_{r=r_{q q}-1}^{N-1} \operatorname{tr}\left(W_{j}\right)\left\|A_{j}^{N-r-1}\right\|_{2}^{2}\right] .
$$


We can then find a small set $\mathcal{D}_{1}$ and $\epsilon_{1}$ such that $f_{1} \geq 1$, and $\Delta V\left(e_{k}, N\right) \leq-f_{1}$, for $e_{k} \notin \mathcal{D}_{1}$, which confirms the conditions in Theorem 1, under the TOD scheduling rule, assuming no packet loss. This proves $f$-ergodicity of $\left\{e_{k}\right\}^{k}$ and consequently of the overall network state $\left(x_{k}, e_{k}\right)$.

\section{A.2 Proof of Theorem 3}

Proof. To take the dropouts into account, re-define the set $G$ to be containing sub-systems which are transmitted, either successful or unsuccessful, at least once over the interval $[k, k+N]$. Assume $|\bar{G}|=m$. According to the law of iterated expectations, and from expressions (A2) and (A4), for a sub-system $i \in G$ with $\delta_{k+r_{i}-1}^{i}=1$, i.e., the latest time a transmission is scheduled, we obtain:

$$
\begin{aligned}
& \mathrm{E}\left[\left\|e_{k+N}^{i}\right\|_{2}^{2} \mid e_{k}, \gamma_{k+r_{i}-1}^{i}\right]=p \mathrm{E}\left[\left\|e_{k+N}^{i}\right\|_{2}^{2} \mid e_{k}, \gamma_{k+r_{i}-1}^{i}=1\right]+(1-p) \mathrm{E}\left[\left\|e_{k+N}^{i}\right\|_{2}^{2} \mid e_{k}, \gamma_{k+r_{i}-1}^{i}=0\right] \\
& \quad \leq p \sum_{r=r_{i}-1}^{N-1} \operatorname{tr}\left(W_{i}\right)\left\|A_{i}^{N-r-1}\right\|_{2}^{2}+(1-p) \mathrm{E}\left[\left\|A_{i}^{N} e_{k}^{i}+\sum_{r=0}^{N-1} A_{i}^{N-r-1} w_{k+r}^{i}\right\|_{2}^{2} \mid e_{k}\right] \\
& \quad \leq p \sum_{r=r_{i}-1}^{N-1} \operatorname{tr}\left(W_{i}\right)\left\|A_{i}^{N-r-1}\right\|_{2}^{2}+(1-p)\left[\left\|A_{i}^{N}\right\|_{2}^{2}\left\|e_{k}^{i}\right\|_{2}^{2}+\sum_{r=0}^{N-1} \operatorname{tr}\left(W_{i}\right)\left\|A_{i}^{N-r-1}\right\|_{2}^{2}\right]
\end{aligned}
$$

If $m>0$, then there exists at least one sub-system $q \in G$ with more than one transmission attempt over $[k, k+N]$. Let time-steps $k+r_{q}$ and $k+r_{q q}$ be defined as in the proof of Theorem 2. This implies $\left\|e_{k+r_{q q}-1}^{q}\right\|_{2}>\left\|e_{k+r_{q q}-1}^{j}\right\|_{2}$ for all $j \in \bar{G}$, according to (9). Recalling (A6), together with the associated dropout probability $1-p$ to each scheduled transmission, we have at time-step $k+r_{q q}-1$

$$
\begin{aligned}
& \mathrm{E}\left[\left\|e_{k+r_{q q}-1}^{q}\right\|_{2}^{2} \mid e_{k}, \gamma_{k+r_{q}-1}^{q}\right]=p \mathrm{E}\left[\left\|e_{k+r_{q q}-1}^{q}\right\|_{2}^{2} \mid e_{k}, \gamma_{k+r_{q}-1}^{q}=1\right]+(1-p) \mathrm{E}\left[\left\|e_{k+r_{q q}-1}^{q}\right\|_{2}^{2} \mid e_{k}, \gamma_{k+r_{q}-1}^{q}=0\right] \\
& \quad \leq p \sum_{r=r_{q}-1}^{r_{q q}-2} \operatorname{tr}\left(W_{q}\right)\left\|A_{q}^{r_{q q}-r-2}\right\|_{2}^{2}+(1-p) \mathrm{E}\left[\left\|A_{q}^{r_{q q}-1} e_{k}^{q}+\sum_{r=0}^{r_{q q}-2} A_{q}^{r_{q q}-r-2} w_{k+r}^{q}\right\|_{2}^{2} \mid e_{k}\right] .
\end{aligned}
$$

Substituting the above expression in (A6), the following upper-bound for $\mathrm{E}\left[\left\|e_{k+N}^{j}\left|\|_{2}^{2}\right| e_{k}\right]\right.$ follows:

$$
\begin{aligned}
\mathrm{E} & {\left[\left\|e_{k+N}^{j}\right\|_{2}^{2} \mid e_{k}\right] \leq p\left\|A_{j}^{N-r_{q q}+1}\right\|_{2}^{2} \sum_{r=r_{q}-1}^{r_{q q}-2} \operatorname{tr}\left(W_{q}\right)\left\|A_{q}^{r_{q q}-r-2}\right\|_{2}^{2} } \\
& +(1-p)\left\|A_{j}^{N-r_{q q}+1}\right\|_{2}^{2} \mathrm{E}\left[\left\|A_{q}^{r_{q q}-1} e_{k}^{q}+\sum_{r=0}^{r_{q q}-2} A_{q}^{r_{q q}-r-2} w_{k+r}^{q}\right\|_{2}^{2} \mid e_{k}\right]+\sum_{r=r_{q q}-1}^{N-1} \operatorname{tr}\left(W_{j}\right)\left\|A_{j}^{N-r-1}\right\|_{2}^{2} \\
& \leq \zeta_{>0}+(1-p)\left\|A_{j}^{N-r_{q q}+1}\right\|_{2}^{2}\left\|A_{q}^{r_{q q}-1}\right\|_{2}^{2}\left\|e_{k}^{q}\right\|_{2}^{2},
\end{aligned}
$$

where, $\zeta_{>0}=p\left\|A_{j}^{N-r_{q q}+1}\right\|_{2}^{2} \sum_{r=r_{q}-1}^{r_{q q}-2} \operatorname{tr}\left(W_{q}\right)\left\|A_{q}^{r_{q q}-r-2}\right\|_{2}^{2}+\sum_{r=r_{q q}-1}^{N-1} \operatorname{tr}\left(W_{j}\right)\left\|A_{j}^{N-r-1}\right\|_{2}^{2}+(1-$ p) $\left\|A_{j}^{N-r_{q q}+1}\right\|_{2}^{2} \sum_{r=0}^{r_{q q}-2} \operatorname{tr}\left(W_{q}\right)\left\|A_{q}^{r_{q q}-r-2}\right\|_{2}^{2}$. Having (A8) and (A9), the $N$-step drift (16) becomes

$$
\begin{aligned}
& \Delta V\left(e_{k}, N\right)=\sum_{i \in G} \mathrm{E}\left[\left\|e_{k+N}^{i}\right\|_{2}^{2} \mid e_{k}\right]+\sum_{j \in \bar{G}} \mathrm{E}\left[\left\|e_{k+N}^{j}\right\|_{2}^{2} \mid e_{k}\right]-V\left(e_{k}\right) \\
& \quad \leq \sum_{i \in G}\left[p \sum_{r=r_{i}-1}^{N-1} \operatorname{tr}\left(W_{i}\right)\left\|A_{i}^{N-r-1}\right\|_{2}^{2}\right]+\sum_{i \in G}\left[(1-p)\left[\left\|A_{i}^{N}\right\|_{2}^{2}\left\|e_{k}^{i}\right\|_{2}^{2}+\sum_{r=0}^{N-1} \operatorname{tr}\left(W_{i}\right)\left\|A_{i}^{N-r-1}\right\|_{2}^{2}\right]\right] \\
& \quad+\sum_{j \in \bar{G}} \zeta_{>0}+(1-p)\left\|A_{j}^{N-r_{q q}+1}\right\|_{2}^{2}\left\|A_{q}^{r_{q q}-1}\right\|_{2}^{2}\left\|e_{k}^{q}\right\|_{2}^{2}-V\left(e_{k}\right) .
\end{aligned}
$$

Then, we can rewrite the $N$-step drift operator $\Delta V\left(e_{k}, N\right)$ as

$$
\Delta V\left(e_{k}, N\right) \leq \tau_{>0}+\sum_{i \in G}\left[(1-p)\left[\left\|A_{i}^{N}\right\|_{2}^{2}\left\|e_{k}^{i}\right\|_{2}^{2}\right]\right]+\sum_{j \in \bar{G}}(1-p)\left\|A_{j}^{N-r_{q q}+1}\right\|_{2}^{2}\left\|A_{q}^{r_{q q}-1}\right\|_{2}^{2}\left\|e_{k}^{q}\right\|_{2}^{2}-V\left(e_{k}\right),
$$


where, $\tau_{>0}=\zeta_{>0}+\sum_{i \in G}\left[p \sum_{r=r_{i}-1}^{N-1} \operatorname{tr}\left(W_{i}\right)\left\|A_{i}^{N-r-1}\right\|_{2}^{2}+(1-p)\left[\sum_{r=0}^{N-1} \operatorname{tr}\left(W_{i}\right)\left\|A_{i}^{N-r-1}\right\|_{2}^{2}\right]\right]$. Considering the fact that $V\left(e_{k}\right)>\left\|e_{k}^{i}\right\|_{2}^{2}$, for every $i \in\{1, \ldots, N\}$, the above expression simplifies to

$$
\Delta V\left(e_{k}, N\right) \leq \tau_{>0}+(1-p) V\left(e_{k}\right)\left[\sum_{j \in \bar{G}}\left\|A_{j}^{N-r_{q q}+1}\right\|_{2}^{2}\left\|A_{q}^{r_{q q}-1}\right\|_{2}^{2}+\sum_{i \in G}\left\|A_{i}^{N}\right\|_{2}^{2}\right]-V\left(e_{k}\right) .
$$

In order to achieve a decreasing drift function, it is essential to satisfy the following inequality:

$$
(1-p)\left[\sum_{j \in \bar{G}}\left\|A_{j}^{N-r_{q q}+1}\right\|_{2}^{2}\left\|A_{q}^{r_{q q}-1}\right\|_{2}^{2}+\sum_{i \in G}\left\|A_{i}^{N}\right\|_{2}^{2}\right]-1<0 .
$$

Considering the worst-case scenario, i.e. $\left\|A_{j}\right\|_{2}>\left\|A_{i}\right\|_{2}$ for all the $m$ sub-systems $j \in \bar{G}$ and $N-m$ sub-systems $i \in G$, the lower bound for the success probability $p$ can be derived as follows

$$
p>1-\frac{1}{\sum_{j \in \bar{G}}\left\|A_{j}\right\|_{2}^{2 N}+\sum_{i \in G}\left\|A_{i}^{N}\right\|_{2}^{2}} .
$$

The above lower bound depends on the time-varying sets $G$ and $\bar{G}$, thus we employ the inequality $\sum_{i=1}^{N}\left\|A_{i}\right\|_{2}^{2 N}>\sum_{j \in \bar{G}}\left\|A_{j}\right\|_{2}^{2 N}+\sum_{i \in G}\left\|A_{i}^{N}\right\|_{2}^{2}$, leading to the time-invariant lower-bound (20).

Having (20) satisfied, we define $f_{2}: \mathbb{R}^{n} \rightarrow \mathbb{R}$ as $f_{2}=\epsilon_{2} V\left(e_{k}\right)-\tau_{>0}$, with $\epsilon_{2} \in(0,1]$. Therefore, small set $\mathcal{D}_{2}$ and $\epsilon_{2}$ can be found such that $f_{2} \geq 1$, and $\Delta V\left(e_{k}, N\right) \leq-f_{2}$, for $e_{k} \notin \mathcal{D}_{2}$. This ensures that the conditions in Theorem 2 hold for the network-induced error Markov chain $\left\{e_{k}\right\}^{k}$ under the TOD scheduling rule, when the lower-bound (20) is satisfied. This guarantees the $f$-ergodicity of estimation error sequence $\left\{e_{k}\right\}^{k}$ and the proof then readily follows.

\section{A.3 Proof of Theorem 4}

Proof. It should be mentioned first that, we search for an analytic and uniform (independent from initial values) upper-bound on the average cost $J_{\text {ave }}$ due to the fact that computing the exact value of $J_{\text {ave }}$, considering the complex form of the event-based TOD scheduler, is generally infeasible. It is proved in (Cogill \& Lall, 2006) that for $J_{e_{k}}: \mathbb{R}^{n} \rightarrow \mathbb{R}$, the following upper-bound exists:

$$
J_{\mathrm{ave}} \leq \sup _{e_{k} \in \mathbb{R}^{n}}\left\{J_{e_{k}}+\mathrm{E}\left[h\left(e_{k+1}\right) \mid e_{k}\right]-h\left(e_{k}\right)\right\}
$$

where, $h: \mathbb{R}^{n} \rightarrow \mathbb{R}$, and $h\left(e_{k}\right) \geq 0$ for all $e_{k} \in \mathbb{R}^{n}$. Recall that we are interested in evaluating the behavior of the Markov process $\left\{e_{k}\right\}^{k}$ over the intervals of length $N$, while (A10) refers to one-step transition of the Lyapunov-like function $h\left(e_{k}\right)$. Due to the fact that $\left\{e_{k}\right\}^{k}$ is a $\psi$-irreducible Markov process evolving in uncountable state space $\mathbb{R}^{n}$, one can always generate a Markov chain that samples the states of the original Markov chain at time instances $\{0, N, 2 N, \ldots\}$. It is straightforward to discuss that $\psi$-irreducibility and aperiodicity of the original Markov process are carried over to the generated chain. Moreover, time-homogeneity of the original Markov chain implies timehomogeneity of the constructed chain (Meyn \& Tweedie, 1996, Chapter 1). Thus, we can replace the original Markov chain $\left\{e_{k}\right\}^{k}$ in (A10) with the generated chain which has the same statistical behavior to that of the original chain. Then the upper-bound (A10) can be re-expressed as follows

$$
J_{\text {ave }} \leq \sup _{e_{k} \in \mathbb{R}^{n}}\left\{J_{e_{k}}+\mathrm{E}\left[h\left(e_{k+N}\right) \mid e_{k}\right]-h\left(e_{k}\right)\right\} .
$$


We introduce the non-negative quadratic function $h\left(e_{k}\right)=\frac{1}{N} \sum_{i=1}^{N}\left\|e_{k}^{i}\right\|_{2}^{2}$. Considering the cost function (21), the upper-bound for the average cost (A11) is therefore reduced to

$$
\begin{aligned}
J_{\text {ave }} & \leq \sup _{e_{k} \in \mathbb{R}^{n}}\left[J_{e_{k}}+\mathrm{E}\left[h\left(e_{k+N}\right) \mid e_{k}\right]-\frac{1}{N} \sum_{i=1}^{N}\left\|e_{k}^{i}\right\|_{2}^{2}\right] \\
& =\sup _{e_{k} \in \mathbb{R}^{n}}\left[\frac{1}{N} \sum_{i=1}^{N}\left\|e_{k}^{i}\right\|_{2}^{2}+\mathrm{E}\left[h\left(e_{k+N}\right) \mid e_{k}\right]-\frac{1}{N} \sum_{i=1}^{N}\left\|e_{k}^{i}\right\|_{2}^{2}\right] \\
& =\sup _{e_{k} \in \mathbb{R}^{n}} \frac{1}{N} \sum_{i=1}^{N} \mathrm{E}\left[\left\|e_{k+N}^{i}\right\|_{2}^{2} \mid e_{k}\right] \\
& =\sup _{e_{k} \in \mathbb{R}^{n}} \frac{1}{|G|} \sum_{i \in G} \mathrm{E}\left[\left\|e_{k+N}^{i}\right\|_{2}^{2} \mid e_{k}\right]+\frac{1}{|\bar{G}|} \sum_{j \in \bar{G}} \mathrm{E}\left[\left\|e_{k+N}^{j}\right\|_{2}^{2} \mid e_{k}\right]
\end{aligned}
$$

To derive the upper-bound for the average cost (A13) with $p=1$, we recall from expressions (A4) and (A7) that the followings hold for sub-systems $i$ in $G$ and sub-systems $j$ in $\bar{G}$, respectively,

$$
\begin{aligned}
& \sum_{i \in G} \mathrm{E}\left[\left\|e_{k+N}^{i}\right\|_{2}^{2} \mid e_{k}^{i}\right] \leq \sum_{i \in G} \sum_{r=r_{i}-1}^{N-1} \operatorname{tr}\left(W_{i}\right)\left\|A_{i}^{N-r-1}\right\|_{2}^{2}, \\
& \sum_{j \in \bar{G}} \mathrm{E}\left[\left\|e_{k+N}^{j}\right\|_{2}^{2} \mid e_{k}\right] \leq \sum_{j \in \bar{G}}\left\|A_{j}^{N-r_{q q}+1}\right\|_{2}^{2} \sum_{r=r_{q}-1}^{r_{q q}-2} \operatorname{tr}\left(W_{q}\right)\left\|A_{q}^{r_{q q}-r-2}\right\|_{2}^{2}+\sum_{j \in \bar{G}} \sum_{r=r_{q q}-1}^{N-1} \operatorname{tr}\left(W_{j}\right)\left\|A_{j}^{N-r-1}\right\|_{2}^{2} .
\end{aligned}
$$

Therefore, we have the following upper-bound for the average cost:

$$
\begin{aligned}
J_{\text {ave }} & \leq \frac{1}{|G|} \sum_{i \in G} \sum_{r=r_{i}-1}^{N-1} \operatorname{tr}\left(W_{i}\right)\left\|A_{i}^{N-r-1}\right\|_{2}^{2} \\
& +\frac{1}{|\bar{G}|} \sum_{j \in \bar{G}}\left\|A_{j}^{N-r_{q q}+1}\right\|_{2}^{2} \sum_{r=r_{q}-1}^{r_{q q}-2} \operatorname{tr}\left(W_{q}\right)\left\|A_{q}^{r_{q q}-r-2}\right\|_{2}^{2}+\sum_{r=r_{q q}-1}^{N-1} \operatorname{tr}\left(W_{j}\right)\left\|A_{j}^{N-r-1}\right\|_{2}^{2},
\end{aligned}
$$

The upper-bound (A14) depends on the varying time instances $r_{q}$ and $r_{q q}$. To have an upper-bound depending solely on constant system parameters, we consider the worst case scenario which entails that for all $m$ sub-systems $j \in \bar{G}$, and all $N-m$ sub-systems $i \in G$, the conditions $\left\|A_{j}\right\|_{2}>\left\|A_{i}\right\|_{2}$, and $W_{j} \succ W_{i}$ hold. Hence, $\left\|A_{j}\right\|_{2}>\left\|A_{q}\right\|_{2}$ and $W_{j} \succ W_{q}$ for any arbitrary $j \in \bar{G}$ and $q \in G$, yields

$$
\begin{aligned}
J_{\text {ave }} & \leq \frac{1}{|G|} \sum_{i \in G} \sum_{r=r_{i}-1}^{N-1} \operatorname{tr}\left(W_{i}\right)\left\|A_{i}^{N-r-1}\right\|_{2}^{2} \\
& +\frac{1}{|\bar{G}|}\left[\sum_{j \in \bar{G}} \sum_{r=r_{q}-1}^{r_{q q}-2} \operatorname{tr}\left(W_{j}\right)\left\|A_{j}^{N-r_{q q}+1}\right\|_{2}^{2}\left\|A_{j}^{r_{q q}-r-2}\right\|_{2}^{2}+\sum_{j \in \bar{G}} \sum_{r=r_{q q}-1}^{N-1} \operatorname{tr}\left(W_{j}\right)\left\|A_{j}^{N-r-1}\right\|_{2}^{2}\right] \\
& \leq \frac{1}{|G|} \sum_{i \in G} \sum_{r=r_{i}-1}^{N-1} \operatorname{tr}\left(W_{i}\right)\left\|A_{i}^{N-r-1}\right\|_{2}^{2} \\
& +\frac{1}{|\bar{G}|} \sum_{j \in \bar{G}} \operatorname{tr}\left(W_{j}\right)\left[\sum_{r=r_{q}-1}^{r_{q q}-2}\left\|A_{j}\right\|_{2}^{2(N-r-1)}+\sum_{r=r_{q q}-1}^{N-1}\left\|A_{j}\right\|_{2}^{2(N-r-1)}\right] \\
& \leq \frac{1}{|G|} \sum_{i \in G} \sum_{r=r_{i}-1}^{N-1} \operatorname{tr}\left(W_{i}\right)\left\|A_{i}^{N-r-1}\right\|_{2}^{2}+\frac{1}{|\bar{G}|} \sum_{j \in \bar{G}} \sum_{r=0}^{N-1} \operatorname{tr}\left(W_{j}\right)\left\|A_{j}\right\|_{2}^{2(N-r-1)} \\
& \leq \sum_{i=1}^{N} \sum_{r=0}^{N-1} \operatorname{tr}\left(W_{i}\right)\left\|A_{i}\right\|_{2}^{2(N-r-1)} .
\end{aligned}
$$

The upper-bound (A15) is uniformly bounded, independent of the initial conditions. 